\title{
Nematic-Isotropic Phase Transition in Liquid Crystals: A Variational Derivation of Effective Geometric Motions
}

\author{
Tim LAUX® \& YUning LiU
}

\author{
Communicated by F. LIN
}

\begin{abstract}
In this work, we study the nematic-isotropic phase transition based on the dynamics of the Landau-De Gennes theory of liquid crystals. At the critical temperature, the Landau-De Gennes bulk potential favors the isotropic phase and nematic phase equally. When the elastic coefficient is much smaller than that of the bulk potential, a scaling limit can be derived by formal asymptotic expansions: the solution gradient concentrates on a closed surface evolving by mean curvature flow. Moreover, on one side of the surface the solution tends to the nematic phase which is governed by the harmonic map heat flow into the sphere while on the other side, it tends to the isotropic phase. To rigorously justify such a scaling limit, we prove a convergence result by combining weak convergence methods and the modulated energy method. Our proof applies as long as the limiting mean curvature flow remains smooth.
\end{abstract}

\section{Introduction}

Nematic liquid crystals react to shear stress like a conventional liquid while the molecules are oriented in a crystal-like way. One of the successful continuum theories modeling nematic liquid crystals is the $Q$-tensor theory, also referred to as Landau-De Gennes theory, which uses a $3 \times 3$ traceless and symmetric matrixvalued function $Q(x)$ as order parameter to characterize the orientation of molecules near a material point $x$ (cf. [8]). The matrix $Q$, also called $Q$-tensor, can be interpreted as the second moment of a number density function

$$
Q(x)=\int_{\mathbb{S}^{2}}\left(p \otimes p-\frac{1}{3} I_{3}\right) f(x, p) \mathrm{d} p,
$$

where $f(x, p)$ corresponds to the number density of liquid crystal molecules which orient along the direction $p \in \mathbb{S}^{2}$ near the material point $x$ (cf. [5]). The configura- 
tion space of the $Q$-tensor is the 5-dimensional linear space

$$
\mathcal{Q}=\left\{Q \in \mathbb{R}^{3 \times 3} \mid Q=Q^{T}, \operatorname{tr} Q=0\right\} .
$$

By elementary linear algebra, each such $Q$ can be written as

$$
Q=s\left(\mathrm{u} \otimes \mathrm{u}-\frac{1}{3} I_{3}\right)+t\left(\mathrm{v} \otimes \mathrm{v}-\frac{1}{3} I_{3}\right)
$$

for some $s, t \in \mathbb{R}$ and $\mathrm{u}, \mathrm{v} \in \mathbb{S}^{2}$ which are perpendicular. In the physics literature, for instance De Gennes-Prost [8], such a representation is called the biaxial nematic configurations, cf. [23]. In case $Q$ has repeated eigenvalues, it is called uniaxial. These $Q$ 's form a 3-dimensional manifold in $\mathcal{Q}$, denoted by

$$
\mathcal{U}:=\left\{Q \in \mathcal{Q} \mid Q=s\left(\mathrm{u} \otimes \mathrm{u}-\frac{1}{3} I_{3}\right) \quad \text { for some } s \in \mathbb{R} \text { and } \mathrm{u} \in \mathbb{S}^{2}\right\},
$$

with a conical singularity at $s=0$. Here the parameter $s$ is called the degree of orientation. To study static configurations of the liquid crystal material in a physical domain $\Omega$, a natural approach is to consider the Ginzburg-Landau type energy

$$
E_{\varepsilon}(Q)=\int_{\Omega}\left(\frac{\varepsilon}{2}|\nabla Q|^{2}+\frac{1}{\varepsilon} F(Q)\right) \mathrm{d} x,
$$

where $\Omega \subset \mathbb{R}^{d}$ is a bounded domain with smooth boundary, $|\nabla Q|=\sqrt{\sum_{i j k}\left|\partial_{k} Q_{i j}\right|^{2}}$, and $F(Q)$ is the bulk energy density

$$
F(Q)=\frac{a}{2} \operatorname{tr}\left(Q^{2}\right)-\frac{b}{3} \operatorname{tr}\left(Q^{3}\right)+\frac{c}{4}\left(\operatorname{tr}\left(Q^{2}\right)\right)^{2} .
$$

Here $a, b, c \in \mathbb{R}^{+}$are material and temperature dependent constants, and $\varepsilon$ denotes the relative intensity of elastic and bulk energy, which is usually quite small. It can be proved that all critical points of $F(Q)$ are uniaxial (1.4), (cf. [23]), and thus

$$
F(Q)=\frac{s^{2}}{27}\left(9 a-2 b s+3 c s^{2}\right)=: f(s), \text { if } Q \text { is uniaxial }(1.4)
$$

Moreover, $F(Q)$ has two families of stable local minimizers corresponding to the following choices of $s=s_{ \pm}$:

$$
s_{-}=0, \quad s_{+}=\frac{b+\sqrt{b^{2}-24 a c}}{4 c} .
$$

In this work we shall consider the bistable case when

$$
b^{2}=27 a c, \quad \text { and } a, c>0 .
$$

By rescaling, one can choose $a=3, b=9, c=1$. From the physics view point, such choices of the coefficients correspond to the critical temperature at which the system favors the nematic phase and the isotropic phase equally $[8$, Section 
2.3]. Analytically, it can be shown that, in this case, the two families of minimizers corresponding to (1.8) are the only global minimizers of $F(Q)$ :

$$
F(Q) \geqq 0 \text { and the equality holds if and only if } Q \in\{0\} \cup \mathcal{N},
$$

where

$$
\begin{aligned}
& \mathcal{N}:=\left\{Q \in \mathcal{Q} \mid Q=s_{+}\left(\mathrm{u} \otimes \mathrm{u}-\frac{1}{3} I_{3}\right) \text { for some } \mathrm{u} \in \mathbb{S}^{2}\right\}, \\
& \text { with } s_{+}=\sqrt{\frac{3 a}{c}} .
\end{aligned}
$$

At this point we digress to mention that the Landau-De Gennes model (1.5) is closely related to Ericksen's model, where the energy is

$$
e_{E}(s, \mathrm{u}):=\int_{\Omega}\left(\kappa|\nabla s|^{2}+s^{2}|\nabla \mathrm{u}|^{2}+\psi(s)\right) \mathrm{d} x .
$$

This model was introduced by Ericksen [9] for the purpose of studying line defects. It can be formally obtained by plugging the uniaxial Ansatz (1.4) into (1.5). In contrast to (1.5) which uses $Q \in \mathcal{Q}$ as order parameter, Ericksen's model uses $(s, \mathrm{u}) \in \mathbb{R} \times \mathbb{S}^{2}$ and is very useful to describe liquid crystal defects. The analysis of this model is very challenging, mainly due to the reason that the geometry of the uniaxial configuration (1.4) corresponds to a double-cone, and the energy (1.12) is highly degenerate when $s=0$. The analytical aspects of such a model have been investigated by many authors, for instance, by Lin [18], Hardt-Lin-Poon [20], Bedford [7], Alper-Hardt-Lin [2], and Alper [1].

To model nematic-isotropic phase transitions in the framework of Landau-De Gennes theory, we shall investigate the small- $\varepsilon$ limit of the natural gradient flow dynamics of (1.5) with initial data undergoing a sharp transition near a smooth interface. To be more precise, we consider the system

$$
\begin{aligned}
\partial_{t} Q_{\varepsilon} & =\Delta Q_{\varepsilon}-\frac{1}{\varepsilon^{2}} \nabla_{q} F\left(Q_{\varepsilon}\right), & & \text { in } \Omega \times(0, T), \\
Q_{\varepsilon}(x, 0) & =Q_{\varepsilon}^{i n}(x), & & \text { in } \Omega, \\
Q_{\varepsilon}(x, t) & =0, & & \text { on } \partial \Omega \times(0, T),
\end{aligned}
$$

where $\nabla_{q} F(Q)$ is the variation of $F(Q)$ in space $\mathcal{Q}$ :

$$
\left(\nabla_{q} F(Q)\right)_{i j}=a Q_{i j}-b \sum_{k=1}^{3} Q_{i k} Q_{k j}+c|Q|^{2} Q_{i j}+\frac{b}{3}|Q|^{2} \delta_{i j} .
$$

The system (1.13a) is the $L^{2}$-gradient flow of energy (1.5) on the slow time scale $\varepsilon$.

Our main result, Theorem 2.1, states that starting from initial conditions with a reasonable nematic-isotropic phase transition from a nematic region $\Omega^{+}(0)$ into an isotropic region $\Omega^{-}(0)$, before the occurrence of topological changes, the solution $Q_{\varepsilon}$ of (1.13) converges to the isotropic phase $Q \equiv 0$ in $\Omega^{-}(t)$ and to a field $Q \in \mathcal{N}$ 
taking values in the nematic phase in $\Omega^{+}(t)$, where the interface between $\Omega^{+}(t)$ and $\Omega^{-}(t)$ moves by mean curvature flow. Furthermore, we show that the limit $Q$ is a harmonic map heat flow from $\Omega^{+}(t)$ into the closed manifold $\mathcal{N}$. Finally, if the region $\Omega^{+}(t)$ is simply-connected, there exists a director field $\mathrm{u}$ such that $Q=s_{+}\left(\mathrm{u} \otimes \mathrm{u}-\frac{1}{3} I_{3}\right), \mathrm{u}$ is a harmonic map heat flow from $\Omega^{+}(t)$ into $\mathbb{S}^{2}$, and satisfies homogenous Neumann boundary conditions on the evolving boundary $\partial \Omega^{+}(t)$.

The proof consists of two key steps: (i) an adaptation of the modulated energy inequality in [12] to the vector-valued case to control the leading-order energy contribution, which is of order $O(1)$ and comes from the phase transition across $\partial \Omega^{+}(t)$. (ii) A version of Chen-Shatah's wedge-product trick in the sense that (1.13) implies

$$
\left[\partial_{t} Q_{\varepsilon}, Q_{\varepsilon}\right]=\nabla \cdot\left[\nabla Q_{\varepsilon}, Q_{\varepsilon}\right]
$$

where $[\cdot, \cdot]$ denotes the commutator.

In (i) we basically follow [12] but need to carefully regularize the metric $d^{F}$ on $Q$ induced by the conformal structure $F(Q)$ in order to exploit the fine properties of its derivative $\nabla_{q} d_{\varepsilon}^{F}$. In particular, we will use the crucial commutator relation $\left[\nabla_{q} d_{\varepsilon}^{F}\left(Q_{\varepsilon}\right), Q_{\varepsilon}\right]=0$ for a.e. $(x, t)$. This seems to lie beyond the realm of generalized chain rules as in [3], which was employed in the work of Simon and one of the authors in [17]. Regarding (ii), we emphasize that the Neumann boundary condition along the free boundary $\partial \Omega^{+}(t)$ can be naturally encoded in the distributional formulation of (1.15) by enlarging the space of test functions. This however, requires uniform $L^{2}$-estimates on the commutators $\left[\partial_{t} Q_{\varepsilon}, Q_{\varepsilon}\right]$ and $\left[\nabla Q_{\varepsilon}, Q_{\varepsilon}\right]$, which are one order of $\varepsilon$ better than the a priori estimates suggest. We show that these estimates are guaranteed by our bounds on the modulated energy.

\section{Main Results}

To state the main result of this work, we assume that

$$
I=\bigcup_{t \in[0, T]}\left(I_{t} \times\{t\}\right) \text { is a smoothly evolving closed surface in } \Omega,
$$

starting from a closed smooth surface $I_{0} \subset \Omega$. Let $\Omega^{+}(t)$ be the domain enclosed by $I_{t}$, and $d\left(x, I_{t}\right)$ be the signed-distance from $x$ to $I_{t}$ which takes positive values in $\Omega^{+}(t)$, and negative values in $\Omega^{-}(t)=\Omega \backslash \overline{\Omega^{+}(t)}$, where

$$
\Omega^{ \pm}(t):=\left\{x \in \Omega \mid d\left(x, I_{t}\right) \gtrless 0\right\} .
$$

Moreover, for each $T>0$ we shall denote the 'distorted' parabolic cylinder by

$$
\Omega_{T}^{ \pm}:=\bigcup_{t \in(0, T)}\left(\Omega^{ \pm}(t) \times\{t\}\right)
$$

For $\delta>0$, the $\delta$-neighborhood of $I_{t}$ is denoted by

$$
I_{t}(\delta):=\left\{x \in \Omega:\left|d\left(x, I_{t}\right)\right|<\delta\right\} .
$$


Thus there exists a sufficiently small number $\delta_{I} \in(0,1)$ such that the nearest point projection $P_{I}(\cdot, t): I_{t}\left(\delta_{I}\right) \rightarrow I_{t}$ is smooth for any $t \in[0, T]$, and the interface (2.1) stays at least $\delta_{I}$ distance away from the boundary of the domain $\partial \Omega$.

To introduce the modulated energy for (1.13), we extend the inner normal vector field $\mathrm{n}_{I}$ of $I_{t}$ to a neighborhood of it by

$$
\xi(x, t):=\eta\left(d\left(x, I_{t}\right)\right) \mathrm{n}_{I}\left(P_{I}(x, t), t\right),
$$

where $\eta$ is a cutoff function satisfying

$$
\begin{aligned}
& \eta \text { is even in } \mathbb{R} \text { and decreases in }[0, \infty) ; \\
& \eta(z)=1-z^{2}, \text { for }|z| \leqq \delta_{I} / 2 ; \quad \eta(z)=0 \text { for }|z| \geqq \delta_{I} .
\end{aligned}
$$

Following $[12,16]$, we define the modulated energy by

$$
E_{\varepsilon}\left[Q_{\varepsilon} \mid I\right](t):=\int_{\Omega}\left(\frac{\varepsilon}{2}\left|\nabla Q_{\varepsilon}(\cdot, t)\right|^{2}+\frac{1}{\varepsilon} F_{\varepsilon}\left(Q_{\varepsilon}(\cdot, t)\right)-\xi \cdot \nabla \psi_{\varepsilon}(\cdot, t)\right) \mathrm{d} x,
$$

where

$$
\begin{aligned}
F_{\varepsilon}(q) & :=F(q)+\varepsilon^{K-1} \text { with } K=4, \\
\psi_{\varepsilon}(x, t) & :=d_{\varepsilon}^{F} \circ Q_{\varepsilon}(x, t), \quad \text { and } d_{\varepsilon}^{F}(q):=\left(\phi_{\varepsilon} * d^{F}\right)(q), \forall q \in \mathcal{Q},
\end{aligned}
$$

and the convolution is understood in the space $\mathcal{Q} \simeq \mathbb{R}^{5}$. Moreover, we set

$$
\phi_{\varepsilon}(q):=\varepsilon^{-5 K} \phi\left(\varepsilon^{-K} q\right),
$$

a family of mollifiers in the 5-dimensional configuration space (1.2). Here $\phi$ is smooth, non-negative, having support in $B_{1}^{\mathcal{Q}}$ (the unit ball in $\mathcal{Q}$ ), and isotropic, i.e. for any orthogonal matrix $R \in O(3)$ and any $q \in \mathcal{Q}$ it holds $\phi\left(R^{T} q R\right)=\phi(q)$. The function $d^{F}$ in (2.8b) is the quasi-distance function

$$
\begin{array}{r}
d^{F}(q):=\inf \left\{\int_{0}^{1} \sqrt{2 F(\gamma(t))}\left|\gamma^{\prime}(t)\right| \mathrm{d} t \mid \gamma \in C^{0,1}([0,1] ; \mathcal{Q}),\right. \\
\gamma(0) \in \mathcal{N}, \gamma(1)=q\},
\end{array}
$$

which was introduced by Sternberg [26] and independently by Tartar-Fonseca [13] for the study of the singular perturbation problem. Some properties of $d^{F}$ are stated in Lemma 3.1 below, and interested readers can find the proof in [19,26]. One can refer to Section 3 for more details of these functions. Throughout, we will assume an $L^{\infty}$-bound of $Q_{\varepsilon}$, i.e.

$$
\left\|Q_{\varepsilon}\right\|_{L^{\infty}(\Omega \times(0, T))} \leqq c_{0}
$$

for some fixed constant $c_{0}$. Such an estimate can be obtained by assuming an uniform $L^{\infty}$-bound of the initial data $Q_{\varepsilon}^{i n}$ and then applying maximum principle to (1.13a), see Lemma 3.3 in the sequel. Note that the choice $K=4$ in (2.8a) is due to a technical reason, and is used in the proof of Lemma 4.1.

The main result of this work is the following: 
Theorem 2.1. Assume the surface $I_{t}$ (2.1) evolves by mean curvature flow and encloses a simply-connected domain $\Omega^{+}(t)$. If the initial datum $Q_{\varepsilon}^{\text {in }}$ of $(1.13)$ is well-prepared in the sense that

$$
E_{\varepsilon}\left[Q_{\varepsilon} \mid I\right](0) \leqq c_{1} \varepsilon
$$

for some constant $c_{1}$ that does not depend on $\varepsilon$, then for some $\varepsilon_{k} \downarrow 0$ as $k \uparrow+\infty$,

$$
\begin{aligned}
& Q_{\varepsilon_{k}} \stackrel{k \rightarrow \infty}{\longrightarrow} Q=s_{ \pm}\left(\mathrm{u}(x, t) \otimes \mathrm{u}(x, t)-\frac{1}{3} I_{3}\right), \\
& \text { strongly in } C\left([0, T] ; L_{l o c}^{2}\left(\Omega^{ \pm}(t)\right)\right),
\end{aligned}
$$

where $s_{ \pm}$are given by (1.8) and

$$
\mathrm{u} \in H^{1}\left(\Omega_{T}^{+} ; \mathbb{S}^{2}\right) .
$$

Moreover, $\mathrm{u}$ is a harmonic map heat flow into $\mathbb{S}^{2}$ with homogenous Neumann boundary conditions in the sense that

$$
\begin{gathered}
\int_{\Omega_{T}^{+}} \partial_{t} \mathrm{u} \wedge \mathrm{u} \cdot \varphi \mathrm{d} x \mathrm{~d} t=-\sum_{j=1}^{d} \int_{\Omega_{T}^{+}} \partial_{j} \mathrm{u} \wedge \mathrm{u} \cdot \partial_{j} \varphi \mathrm{d} x \mathrm{~d} t \\
\forall \varphi \in C^{1}\left(\bar{\Omega} \times[0, T] ; \mathbb{R}^{3}\right),
\end{gathered}
$$

where $\wedge$ is the wedge product in $\mathbb{R}^{3}$.

Remark 2.2. Note that (2.15) encodes both the harmonic map heat flow into $\mathbb{S}^{2}$ and the boundary conditions. Indeed, if $\left(\partial_{t} u, \nabla^{2} u\right)$ is continuous up to the boundary of $\Omega^{+}(t)$, then the weak formulation (2.15) implies that $\mathrm{u}$ is a harmonic heat flow into $\mathbb{S}^{2}$ with Neumann boundary conditions on $I_{t}$ :

$$
\partial_{t} \mathrm{u}=\Delta \mathrm{u}+|\nabla \mathrm{u}|^{2} \mathrm{u} \text { in } \Omega_{T}^{+}, \quad \partial_{\mathrm{n}_{I}} \mathrm{u}=0 \text { on } \bigcup_{t \in(0, T)}\left(I_{t} \times\{t\}\right)
$$

If $\Omega^{+}(t)$ is multi-connected, for instance when $\Omega^{+}(t)$ is the region outside $I_{t}$, then a well-known orientability issue arises and the conclusion (2.14) usually only holds away from defects. See the work of Bedford [7] for more discussions of such issues.

Theorem 2.1 solves a special case of the Keller-Rubinstein-Sternberg problem [25] using the energy method. A similar result has been established previously by Fei et al. $[10,11]$ using matched asymptotic expansions and spectral gap estimates. Our approach has the superiority that it allows more flexible initial data, as indicated by Proposition 2.3 below. The general case of the Keller-Rubinstein-Sternberg problem is fairly sophisticated and remains open. We refer the interested readers to a recent work of Lin-Wang [21] for the well-posedness of the limiting system. On the other hand, the static problem has been investigated by Lin et al. [19]. It is worthy to mention that recently Golovaty et al. $[14,15]$ studied a model problem based on highly disparate elastic constants. Most recently, Lin-Wang [22] studied isotropic-nematic transitions based on an anisotropic Ericksen's model. 
Now we turn to the construction of initial data $Q_{\varepsilon}^{i n}$ satisfying (2.12). Let $I_{0} \subset \Omega$ be a smooth closed surface and let $I_{0}\left(\delta_{0}\right)$ be a neighborhood in which the signed distance function $d\left(x, I_{0}\right)$ is smooth. Let $\zeta(z)$ be a cut-off function such that

$$
\zeta(z)=0 \text { for }|z| \geqq 1 \text {, and } \zeta(z)=1 \text { for }|z| \leqq 1 / 2 \text {. }
$$

Then we define

$$
\tilde{S}_{\varepsilon}(x):=\zeta\left(\frac{d\left(x, I_{0}\right)}{\delta_{0}}\right) S\left(\frac{d\left(x, I_{0}\right)}{\varepsilon}\right)+\left(1-\zeta\left(\frac{d\left(x, I_{0}\right)}{\delta_{0}}\right)\right) s_{+} \mathbf{1}_{\Omega^{+}(0)},
$$

where $S(z)$ is given by the optimal profile

$$
S(z):=\frac{s_{+}}{2}\left(1+\tanh \left(\frac{\sqrt{a}}{2} z\right)\right), \quad z \in \mathbb{R}
$$

Note that (2.19) is the solution of the ODE

$$
-S^{\prime \prime}(z)+a S(z)-\frac{b}{3} S^{2}(z)+\frac{2}{3} c S^{3}(z)=0, S(-\infty)=0, S(+\infty)=s_{+} .
$$

Proposition 2.3. For every $\mathrm{u}^{\text {in }} \in H^{1}\left(\Omega ; \mathbb{S}^{2}\right)$, the initial datum defined by

$$
Q_{\varepsilon}^{i n}(x):=\tilde{S}_{\varepsilon}(x)\left(\mathrm{u}^{i n}(x) \otimes \mathrm{u}^{i n}(x)-\frac{1}{3} I_{3}\right)
$$

satisfies $Q_{\varepsilon}^{i n} \in H^{1}(\Omega ; \mathcal{Q}) \cap L^{\infty}(\Omega ; \mathcal{Q})$ and

$$
Q_{\varepsilon}^{i n}(x)=\left\{\begin{aligned}
s_{+}\left(\mathrm{u}^{i n} \otimes \mathrm{u}^{i n}-\frac{1}{3} I_{3}\right) & \text { if } x \in \Omega^{+}(0) \backslash I_{0}\left(\delta_{0}\right), \\
S\left(\frac{d\left(x, I_{0}\right)}{\varepsilon}\right)\left(\mathrm{u}^{i n} \otimes \mathrm{u}^{i n}-\frac{1}{3} I_{3}\right) & \text { if } x \in I_{0}\left(\delta_{0} / 2\right), \\
0 & \text { if } x \in \Omega^{-}(0) \backslash I_{0}\left(\delta_{0}\right) .
\end{aligned}\right.
$$

Moreover, there exists a constant $c_{1}>0$ which only depends on $I_{0}$ and $\left\|\mathrm{u}^{i n}\right\|_{H^{1}(\Omega)}$ such that $Q_{\varepsilon}^{i n}$ is well-prepared in the sense of (2.12).

The rest of this work will be organized as follows: in Section 3 we discuss some properties of the quasi-distance function (2.10) and use them to construct the well-prepared initial data (2.21) and thus prove Proposition 2.3. In Section 4 we establish a relative-entropy type inequality for the parabolic system (1.13). Based on the various estimates given by such an inequality, in Section 5 we study the limit $\varepsilon \downarrow 0$ of (1.13) and give the proof of Theorem 2.1. 


\section{Preliminaries}

We start with a lemma about the quasi-distance function (2.10), which was originally due to $[13,26]$.

Lemma 3.1. The function $d^{F}(q)$ is locally Lipschitz in $\mathcal{Q}$ with point-wise derivative satisfying

$$
\left|\nabla_{q} d^{F}(q)\right|=\sqrt{2 F(q)} \text { for a.e. } q \in \mathcal{Q} \text {. }
$$

Moreover,

$$
d^{F}(q)= \begin{cases}0 & \text { if } q \in \mathcal{N} \\ c^{F} & \text { if } q=0,\end{cases}
$$

where $c^{F}$ is the 1-d energy of the minimal connection between $\mathcal{N}$ and 0 :

$$
\begin{array}{r}
c^{F}:=\inf \left\{\int _ { 0 } ^ { 1 } \left(\frac{\left|\gamma^{\prime}(t)\right|^{2}}{2}+F(\gamma(t)) \mathrm{d} t \mid \gamma \in C^{0,1}([0,1] ; \mathcal{Q}),\right.\right. \\
\gamma(0) \in \mathcal{N}, \gamma(1)=0\} .
\end{array}
$$

By elementary linear algebra, any $Q \in \mathcal{Q}$ can be expressed by $Q$ $=\sum_{i=1}^{3} \lambda_{i}(Q) P_{i}(Q)$ with $\sum_{i=1}^{3} \lambda_{i}(Q)=0$, where $P_{i}(Q)=\mathrm{n}_{i} \otimes \mathrm{n}_{i}$ denotes the projection onto the $i$-th eigenspace, and $\lambda_{1}(Q) \leqq \lambda_{2}(Q) \leqq \lambda_{3}(Q)$ are the eigenvalues ordered increasingly. Furthermore using the identities $\sum_{j=1}^{3} \lambda_{j}(Q)=0$ and $I_{3}=\sum_{j=1}^{3} P_{j}(Q)$, we may write

$$
\begin{aligned}
& Q=\left(s+\frac{r}{3}\right)\left(P_{3}(Q)-\frac{1}{3} I_{3}\right)+\frac{2 r}{3}\left(P_{2}(Q)-\frac{1}{3} I_{3}\right), \\
& \text { where } s(Q)=\frac{3}{2} \lambda_{3}(Q), r(Q)=\frac{3}{2}\left(\lambda_{2}(Q)-\lambda_{1}(Q)\right) .
\end{aligned}
$$

The next lemma gives a precise form of $d^{F}$ for uniaxial $Q$-tensors.

Lemma 3.2. If $Q=s_{0}\left(\mathrm{u} \otimes \mathrm{u}-\frac{1}{3} I_{3}\right)$ for some $s_{0} \in\left[0, s_{+}\right]$and $\mathrm{u} \in \mathbb{S}^{2}$, then

$$
d^{F}(Q)=\frac{2}{\sqrt{3}} \int_{s_{0}}^{s_{+}} \sqrt{f(\tau)} \mathrm{d} \tau=: g\left(s_{0}\right),
$$

where $f(s)$ is given by (1.7).

Proof. The argument here is similar to that in [24]. Let $\gamma$ be any curve connecting $\mathcal{N}$ to $Q$. When expressed in the form of eigenframe $\gamma(t)=\sum_{i=1}^{3} \lambda_{i}(t) \mathrm{n}_{i}(t) \otimes \mathrm{n}_{i}(t)$, we claim that $\mathrm{n}_{i}$ are constant in $t$. Actually using the identity

$$
\lambda_{i}^{2}\left|\mathrm{n}_{i}^{\prime}\right|^{2}=\lambda_{i}^{2} \sum_{j=1}^{3}\left(\mathrm{n}_{j} \cdot \mathrm{n}_{i}^{\prime}\right)^{2}=\lambda_{i}^{2} \sum_{j \neq i}\left(\mathrm{n}_{j} \cdot \mathrm{n}_{i}^{\prime}\right)^{2},
$$


we calculate

$$
\begin{aligned}
& \left|\gamma^{\prime}\right|^{2}=\left(\lambda_{1}^{\prime}\right)^{2}+\left(\lambda_{2}^{\prime}\right)^{2}+\left(\lambda_{3}^{\prime}\right)^{2}+2 \sum_{i=1}^{3} \lambda_{i}^{2}\left|\mathrm{n}_{i}^{\prime}\right|^{2} \\
& \quad+\sum_{k=1}^{3} \sum_{1 \leqq i<j \leqq 3} 4 \lambda_{i} \lambda_{j}\left(\mathrm{n}_{i} \cdot \mathrm{n}_{j}^{\prime}\right)\left(\mathrm{n}_{j} \cdot \mathrm{n}_{i}^{\prime}\right) \\
& =\left(\lambda_{1}^{\prime}\right)^{2}+\left(\lambda_{2}^{\prime}\right)^{2}+\left(\lambda_{3}^{\prime}\right)^{2}+\sum_{k=1}^{3} \sum_{1 \leqq i<j \leqq 3} 2\left(\lambda_{i}\left(\mathrm{n}_{j} \cdot \mathrm{n}_{i}^{\prime}\right)+\lambda_{j}\left(\mathrm{n}_{i} \cdot \mathrm{n}_{j}^{\prime}\right)\right)^{2} \\
& \geqq\left(\lambda_{1}^{\prime}\right)^{2}+\left(\lambda_{2}^{\prime}\right)^{2}+\left(\lambda_{3}^{\prime}\right)^{2} .
\end{aligned}
$$

This implies that the global minimum is achieved by a path $\gamma(t)$ with constant eigenframe. So by (3.4) we may write

$$
\begin{array}{r}
\gamma(t)=\operatorname{diag}\left\{-\frac{s(t)+r(t)}{3},-\frac{s(t)-r(t)}{3}, \frac{2 s(t)}{3}\right\}, \\
\text { with }\left.(s, r)\right|_{t=0}=\left(s_{+}, 0\right),\left.\quad(s, r)\right|_{t=1}=\left(s_{0}, 0\right) .
\end{array}
$$

then by (1.6)

$$
F(\gamma(t))=\frac{a}{9}\left(3 s^{2}+r^{2}\right)+\frac{c}{81}\left(3 s^{2}+r^{2}\right)^{2}-\frac{2 b}{27}\left(s^{3}-s r^{2}\right)=: \tilde{F}(r, s) .
$$

Writing $\sqrt{3} s+i r=: \rho e^{i \theta}$ with $i=\sqrt{-1}$, we have $3 \sqrt{3}\left(s^{3}-s r^{2}\right)=\rho^{3} \cos 3 \theta$, and thus

$$
\begin{aligned}
& \int_{0}^{1}\left|\gamma^{\prime}(t)\right| \sqrt{2 F(\gamma(t))} \mathrm{d} t \\
= & \frac{2}{3} \int_{0}^{1} \sqrt{3 s^{\prime 2}+r^{\prime 2}} \sqrt{\tilde{F}(s, r)} \mathrm{d} t \\
= & \frac{2}{3} \int_{0}^{1} \sqrt{\rho^{\prime 2}+\rho^{2} \theta^{\prime 2}} \sqrt{\frac{a \rho^{2}}{9}+\frac{c \rho^{4}}{81}-\frac{2 b \rho^{3}}{81 \sqrt{3}} \cos 3 \theta} \mathrm{d} t .
\end{aligned}
$$

It is clear that this energy is minimized when $\theta \equiv 0$, which corresponds to the uniaxial solution $r(t) \equiv 0$. In view of (1.7)

$$
\int_{0}^{1}\left|\gamma^{\prime}(t)\right| \sqrt{2 F(\gamma(t))} \mathrm{d} t \geqq \frac{2}{\sqrt{3}} \int_{0}^{1}\left|s^{\prime}(t)\right| \sqrt{f(s(t))} \mathrm{d} t .
$$

One can verify that the minimum of the right hand side can be achieved by a monotone function $s(t)$, and thus (3.6) follows from a change of variable.

At this point we would like to remark that for the general Keller-RubinsteinSternberg problem it is very hard to obtain a precise form of $d^{F}$ like (3.6) (cf. [26, Part 2, Lemmas 5 and 7]). 
Before giving the proof of Proposition 2.3, we digress here and discuss the convolution in (2.8b). The space $\mathcal{Q}$ (1.2) is equipped with the inner product $A: B=$ $\operatorname{tr} A^{T} B$, and one can easily verify that $\left\{E_{i}\right\}_{i=1}^{5}$ defined below form an orthonormal basis:

$$
\begin{aligned}
& E_{1}=\left[\begin{array}{ccc}
\frac{\sqrt{3}-3}{6} & 0 & 0 \\
0 & \frac{\sqrt{3}+3}{6} & 0 \\
0 & 0 & -\frac{\sqrt{3}}{3}
\end{array}\right], \quad E_{2}=\left[\begin{array}{ccc}
\frac{\sqrt{3}+3}{6} & 0 & 0 \\
0 & \frac{\sqrt{3}-3}{6} & 0 \\
0 & 0 & -\frac{\sqrt{3}}{3}
\end{array}\right], \\
& E_{3}=\left[\begin{array}{ccc}
0 & \frac{\sqrt{2}}{2} & 0 \\
\frac{\sqrt{2}}{2} & 0 & 0 \\
0 & 0 & 0
\end{array}\right], \quad E_{4}=\left[\begin{array}{ccc}
0 & 0 & \frac{\sqrt{2}}{2} \\
0 & 0 & 0 \\
\frac{\sqrt{2}}{2} & 0 & 0
\end{array}\right], \quad E_{5}=\left[\begin{array}{ccc}
0 & 0 & 0 \\
0 & 0 & \frac{\sqrt{2}}{2} \\
0 & \frac{\sqrt{2}}{2} & 0
\end{array}\right] .
\end{aligned}
$$

This establishes an isometry $\mathcal{Q} \simeq \mathbb{R}^{5}$ and thus the convolution operation in (2.8b) can be interpreted as an integration in $\mathbb{R}^{5}$. Concerning the choice of $\phi$ in (2.9), one can simply choose $g \in C_{c}^{\infty}(\mathbb{R})$ and set $\phi(q):=g\left(\operatorname{tr}\left(q^{2}\right)\right)$, which is obviously isotropic in $q$.

Proof of Proposition 2.3. As a consequence of the choice of the cutoff function $\zeta$ satisfying (2.17), we deduce that (2.22) is fulfilled and $\tilde{S}_{\varepsilon}$ is smooth. To compute the modulated energy (2.7) of the initial data $Q_{\varepsilon}^{i n}$, we write (2.18) by

$$
\tilde{S}_{\varepsilon}(x)=S\left(\frac{d\left(x, I_{0}\right)}{\varepsilon}\right)+\hat{S}_{\varepsilon}(x),
$$

where

$$
\hat{S}_{\varepsilon}(x):=\left(1-\zeta\left(\frac{d\left(x, I_{0}\right)}{\delta_{0}}\right)\right)\left(s_{+} \mathbf{1}_{\Omega^{+}(0)}-S\left(\frac{d\left(x, I_{0}\right)}{\varepsilon}\right)\right) .
$$

It follows from the exponential decay of (2.19) that

$$
\left\|\hat{S}_{\varepsilon}\right\|_{L^{\infty}(\Omega)}+\left\|\nabla \hat{S}_{\varepsilon}\right\|_{L^{\infty}(\Omega)} \leqq C e^{-\frac{C}{\varepsilon}},
$$

for some constant $C>0$ that only depends on $I_{0}$. So we can write

$$
\left|\nabla Q_{\varepsilon}^{i n}\right|^{2}=\frac{2}{3 \varepsilon^{2}} S^{\prime 2}+2 S^{2}\left|\nabla \mathrm{u}^{i n}\right|^{2}+O\left(e^{-C / \varepsilon}\right)\left(\left|\nabla \mathrm{u}^{i n}\right|^{2}+1\right)
$$

Recalling the form of the bulk energy (1.7) for uniaxial $Q$-tensors, in view of (1.9), we have

$$
f(s)=\frac{c}{9} s^{2}\left(s-s_{+}\right)^{2}, \quad \sqrt{f(s)}=\frac{\sqrt{c}}{3} s\left|s-s_{+}\right|, \text {for all } s \in\left[0, s_{+}\right],
$$

and $F\left(Q_{\varepsilon}^{i n}\right)=f\left(S+\hat{S}_{\varepsilon}\right)$. Thus the integrand of $E_{\varepsilon}\left[Q_{\varepsilon} \mid I\right](0)$ can be written as

$$
\begin{aligned}
& \frac{\varepsilon}{2}\left|\nabla Q_{\varepsilon}^{i n}\right|^{2}+\frac{1}{\varepsilon} F\left(Q_{\varepsilon}^{i n}\right)-\frac{2 S^{\prime}}{\varepsilon} \sqrt{\frac{f(S)}{3}} \\
= & \frac{S^{\prime 2}}{3 \varepsilon}+\frac{f(S)}{\varepsilon}-\frac{2 S^{\prime}}{\varepsilon} \sqrt{\frac{f(S)}{3}} \\
& +\varepsilon S^{2}\left|\nabla \mathrm{u}^{i n}\right|^{2}+O\left(e^{-C / \varepsilon}\right)\left(\left|\nabla \mathrm{u}^{i n}\right|^{2}+1\right)+\frac{f\left(S+\hat{S}_{\varepsilon}\right)-f(S)}{\varepsilon} .
\end{aligned}
$$


The first line on the right hand side vanishes due to the identity $S^{\prime}(z)=\sqrt{3 f(S(z))}$ as a consequence of (2.19) or equivalently the ODE (2.20):

$$
\begin{aligned}
& \frac{\varepsilon}{2}\left|\nabla Q_{\varepsilon}^{i n}\right|^{2}+\frac{1}{\varepsilon} F\left(Q_{\varepsilon}^{i n}\right)-\frac{2 S^{\prime}}{\varepsilon} \sqrt{\frac{f(S)}{3}} \\
= & \varepsilon S^{2}\left|\nabla \mathrm{u}^{i n}\right|^{2}+O\left(e^{-C / \varepsilon}\right)\left(\left|\nabla \mathrm{u}^{i n}\right|^{2}+1\right)+\frac{f\left(S+\hat{S}_{\varepsilon}\right)-f(S)}{\varepsilon} .
\end{aligned}
$$

On the other hand, since $0 \leqq \tilde{S}_{\varepsilon} \leqq s_{+}$, by Lemma 3.2,

$$
d^{F}\left(Q_{\varepsilon}^{i n}(x)\right)=\frac{2}{\sqrt{3}} \int_{\tilde{S}_{\varepsilon}(x)}^{s_{+}} \sqrt{f(\tau)} \mathrm{d} \tau .
$$

This together with (2.6) and (3.15) implies

$$
\begin{aligned}
& -(\xi \cdot \nabla) d^{F}\left(Q_{\varepsilon}^{i n}\right) \\
= & -\eta\left(d\left(x, I_{0}\right)\right) \frac{2 S^{\prime}}{\sqrt{3} \varepsilon} \sqrt{f(S)}-\xi \cdot \mathrm{n}_{I} \frac{2 S^{\prime}}{\sqrt{3} \varepsilon}\left(\sqrt{f(S)}-\sqrt{f\left(S+\hat{S}_{\varepsilon}\right)}\right)+O\left(e^{-C / \varepsilon}\right) .
\end{aligned}
$$

Adding up (3.19) and (3.21) yields

$$
\begin{aligned}
& \frac{\varepsilon}{2}\left|\nabla Q_{\varepsilon}^{i n}\right|^{2}+\frac{1}{\varepsilon} F\left(Q_{\varepsilon}^{i n}\right)-(\xi \cdot \nabla) d^{F}\left(Q_{\varepsilon}^{i n}\right) \\
= & \varepsilon S^{2}\left|\nabla \mathrm{u}^{i n}\right|^{2}+O\left(e^{-C / \varepsilon}\right)\left(\left|\nabla \mathrm{u}^{i n}\right|^{2}+1\right)+\frac{f\left(S+\hat{S}_{\varepsilon}\right)-f(S)}{\varepsilon} \\
& +\left(1-\eta\left(d\left(x, I_{0}\right)\right)\right) \frac{2 S^{\prime}}{\varepsilon} \sqrt{\frac{f(S)}{3}}-\xi \cdot \mathrm{n}_{I} \frac{2 S^{\prime}}{\varepsilon}\left(\sqrt{\frac{f(S)}{3}}-\sqrt{\frac{f\left(S+\hat{S}_{\varepsilon}\right)}{3}}\right) .
\end{aligned}
$$

By the exponential decay of (2.19) and (3.15),

$$
\begin{aligned}
& \frac{\varepsilon\left|\nabla Q_{\varepsilon}^{i n}\right|^{2}}{2}+\frac{F\left(Q_{\varepsilon}^{i n}\right)}{\varepsilon}-(\xi \cdot \nabla) d^{F}\left(Q_{\varepsilon}^{i n}\right) \\
\leqq & \varepsilon S^{2}\left|\nabla \mathrm{u}^{i n}\right|^{2}+O\left(e^{-C / \varepsilon}\right)\left(\left|\nabla \mathrm{u}^{i n}\right|^{2}+1\right)+\left(1-\eta\left(d\left(x, I_{0}\right)\right)\right) \frac{2 S^{\prime}}{\varepsilon} \sqrt{\frac{f(S)}{3}}
\end{aligned}
$$

To treat the last term, we first deduce from the exponential decay of (2.19) that

$$
\left\|\left(\frac{d\left(x, I_{0}\right)}{\varepsilon}\right)^{2} S^{\prime}\left(\frac{d\left(x, I_{0}\right)}{\varepsilon}\right)\right\|_{L^{\infty}\left(I_{0}\left(\delta_{0}\right)\right)} \leqq C
$$


for some $C$ that only depends on $I_{0}$. This together with (2.6) implies

$$
\begin{aligned}
& \left(1-\eta\left(d\left(x, I_{0}\right)\right)\right) \frac{2 S^{\prime}}{\varepsilon} \sqrt{\frac{f(S)}{3}} \\
= & \varepsilon^{2} \frac{d^{2}\left(x, I_{0}\right)}{\varepsilon^{2}} \frac{2 S^{\prime}}{\varepsilon} \sqrt{\frac{f(S)}{3}}-\eta\left(d\left(x, I_{0}\right)\right) \mathbf{1}_{\left\{d\left(x, I_{0}\right)>\delta_{0} / 2\right\}} \frac{2 S^{\prime}}{\varepsilon} \sqrt{\frac{f(S)}{3}} \\
\leqq & \varepsilon C\left(I_{0}\right)+C e^{-\frac{C}{\varepsilon}} .
\end{aligned}
$$

Substituting the above estimate into (3.23) and use (2.8a), we arrive at

$$
\begin{aligned}
& \int_{\Omega}\left(\frac{\varepsilon}{2}\left|\nabla Q_{\varepsilon}^{i n}\right|^{2}+\frac{1}{\varepsilon} F_{\varepsilon}\left(Q_{\varepsilon}^{i n}\right)-(\xi \cdot \nabla) d^{F}\left(Q_{\varepsilon}^{i n}\right)\right) \mathrm{d} x \\
& \leqq\left(\varepsilon+O\left(e^{-C / \varepsilon}\right)\right) \int_{\Omega}\left|\nabla \mathrm{u}^{i n}\right|^{2} \mathrm{~d} x+\varepsilon^{2}|\Omega| .
\end{aligned}
$$

On the other hand, by (2.9) and (3.1), we have

$$
\left|d_{\varepsilon}^{F}(q)-d^{F}(q)\right|=\left|\left(\phi_{\varepsilon} * d^{F}\right)(q)-d^{F}(q)\right| \leqq \varepsilon L, \text { if }|q| \leqq M,
$$

where $L=L(M, \phi, F)$. This pointwise estimate implies

$$
\begin{aligned}
& \left|\int_{\Omega}\left((\xi \cdot \nabla) d^{F}\left(Q_{\varepsilon}^{i n}\right)-(\xi \cdot \nabla) d_{\varepsilon}^{F}\left(Q_{\varepsilon}^{i n}\right)\right) \mathrm{d} x\right| \\
& \quad=\left|\int_{\Omega}(\nabla \cdot \xi)\left(d^{F}\left(Q_{\varepsilon}^{i n}\right)-d_{\varepsilon}^{F}\left(Q_{\varepsilon}^{i n}\right)\right) \mathrm{d} x\right| \leqq L \varepsilon,
\end{aligned}
$$

which together with (3.26) implies (2.12).

The next result is concerned with a maximum modulus estimate of (1.13a).

Lemma 3.3. Assume $Q_{\varepsilon}$ is the solution of (1.13) satisfying $\left\|Q_{\varepsilon}^{i n}\right\|_{L^{\infty}(\Omega)} \leqq C_{0}$ for some fixed constant $C_{0}$. Then there exists an $\varepsilon$-independent constant $c_{0}=$ $c_{0}\left(a, b, c, C_{0}\right)>0$ such that

$$
\left\|Q_{\varepsilon}\right\|_{L^{\infty}(\Omega \times(0, T))} \leqq c_{0} .
$$

Proof. On the one hand, by (1.13a), $\left|Q_{\varepsilon}\right|^{2}$ fulfills the following identity

$$
\partial_{t}\left|Q_{\varepsilon}\right|^{2}-\Delta\left|Q_{\varepsilon}\right|^{2}+\left|\nabla Q_{\varepsilon}\right|^{2}=-\frac{2}{\varepsilon^{2}}\left(a\left|Q_{\varepsilon}\right|^{2}-b \operatorname{tr} Q_{\varepsilon}^{3}+c\left|Q_{\varepsilon}\right|^{4}\right) .
$$

On the other hand, there exists $\mu>0$ (sufficiently large) such that $|Q| \geqq \mu$ implies

$$
a|Q|^{2}-b \operatorname{tr} Q^{3}+c|Q|^{4}>0 .
$$

Assume $\left|Q_{\varepsilon}\right|(x, t)$ achieves its maximum at $\left(x_{\varepsilon}, t_{\varepsilon}\right) \in \overline{\Omega \times(0, T)}$. If $\left|Q_{\varepsilon}\left(x_{\varepsilon}, t_{\varepsilon}\right)\right| \leqq$ $\mu$, then we obtain the desired estimate. Otherwise there holds $\partial_{t}\left|Q_{\varepsilon}\right|^{2}-\Delta\left|Q_{\varepsilon}\right|^{2} \leqq 0$, and the weak maximum principle implies the maximum must be achieved on the parabolic boundary $(\partial \Omega \times(0, T)) \cup(\Omega \times\{0\})$, on which $\left|Q_{\varepsilon}\right|$ is bounded by our assumptions. 


\section{The Modulated Energy Inequality}

As the gradient flow of (1.5), the system (1.13a) has the following energy dissipation law

$$
E_{\varepsilon}\left(Q_{\varepsilon}(\cdot, T)\right)+\int_{0}^{T} \int_{\Omega} \varepsilon\left|\partial_{t} Q_{\varepsilon}\right|^{2} \mathrm{~d} x \mathrm{~d} t=E_{\varepsilon}\left(Q_{\varepsilon}^{i n}(\cdot)\right), \text { for all } T \geqq 0 .
$$

Due to the concentration of $\nabla Q_{\varepsilon}$ near the interface $I_{t}$, this estimate is not sufficient to derive the convergence of $Q_{\varepsilon}$. Following a recent work of Fisher et al. [12] we shall develop in this section a calibrated inequality, which modulates the surface energy.

Recall in (2.5) that we extend the normal vector field $\mathrm{n}_{I}$ of the interface $I_{t}$ to a neighborhood of it. We also extend the mean curvature vector $\mathrm{H}_{I}$ of (2.1) to a neighborhood by

$$
\begin{aligned}
& \mathrm{H}_{I}(x, t)=\tilde{\eta}\left(d\left(x, I_{t}\right)\right) \mathrm{H}_{I}\left(P_{I}(x, t), t\right) \\
& \quad=\tilde{\eta}\left(d\left(x, I_{t}\right)\right)\left(\nabla \cdot \mathrm{n}_{I}\right)\left(P_{I}(x, t), t\right) \mathrm{n}_{I}\left(P_{I}(x, t), t\right),
\end{aligned}
$$

where $\tilde{\eta} \in C_{c}^{\infty}\left(\left(-\delta_{I}, \delta_{I}\right)\right)$ is a cut-off which is identically equal to 1 for $s \in$ $\left(-\delta_{I} / 2, \delta_{I} / 2\right)$, and $P_{I}(x, t)=x-\nabla d\left(x, I_{t}\right) d\left(x, I_{t}\right)$ is the projection onto $I_{t}$. The definitions (2.5) and (4.2) of $\xi$ and $\mathrm{H}_{I}$, respectively, imply the following relations:

$$
\begin{aligned}
\partial_{t} \xi & =-\left(\mathrm{H}_{I} \cdot \nabla\right) \xi-\left(\nabla \mathrm{H}_{I}\right)^{T} \xi+O\left(d\left(x, I_{t}\right)\right), \\
\partial_{t}|\xi|^{2} & =-\left(\mathrm{H}_{I} \cdot \nabla\right)|\xi|^{2}+O\left(d^{2}\left(x, I_{t}\right)\right),
\end{aligned}
$$

where $\nabla \mathrm{H}_{I}:=\left\{\partial_{j}\left(\mathrm{H}_{I}\right)_{i}\right\}_{1 \leq i, j \leq d}$ is a matrix with $i$ being the row index. Actually in $I_{t}\left(\delta_{I} / 2\right)$ there holds $\partial_{t} d\left(x, I_{t}\right)=-\mathrm{n}_{I} \cdot \mathrm{H}_{I}\left(P_{I}(x, t)\right)$ and $\nabla d\left(x, I_{t}\right)=\mathrm{n}_{I}\left(P_{I}(x, t)\right)$. So we obtain (4.3) by chain rule. Moreover,

$$
-\nabla \cdot \xi=\mathrm{H}_{I} \cdot \xi+O\left(d\left(x, I_{t}\right)\right),
$$

and since $\mathrm{H}_{I}$ is extended constantly in normal direction, we have

$$
(\xi \cdot \nabla) \mathrm{H}_{I}=0 \text { for all }(x, t) \text { such that }\left|d\left(x, I_{t}\right)\right|<\delta_{I} / 2 .
$$

Moreover, by the choice of $\delta_{I}$ at the beginning of Section 2, we have

$$
\xi=0 \text { on } \partial \Omega \text { and } \mathrm{H}_{I}=0 \text { on } \partial \Omega .
$$

Finally, we have the following regularity

$$
|\nabla \xi|+\left|\mathrm{H}_{I}\right|+\left|\nabla \mathrm{H}_{I}\right| \leqq C\left(I_{0}\right) .
$$

We denote the phase-field analogs of the mean curvature and normal vectors by

$$
\begin{aligned}
\mathrm{H}_{\varepsilon}(x, t) & :=-\left(\varepsilon \Delta Q_{\varepsilon}-\frac{\nabla_{q} F\left(Q_{\varepsilon}\right)}{\varepsilon}\right): \frac{\nabla Q_{\varepsilon}}{\left|\nabla Q_{\varepsilon}\right|}, \\
\mathrm{n}_{\varepsilon}(x, t) & :=\frac{\nabla \psi_{\varepsilon}(x, t)}{\left|\nabla \psi_{\varepsilon}(x, t)\right|},
\end{aligned}
$$


respectively, where $\psi_{\varepsilon}$ is defined by (2.8b). Here and throughout we use the convention that : denotes the contraction in the indices $i, j$ in three-tensors like $\partial_{k} Q_{i, j}$, i.e., the scalar product in the state space $\mathcal{Q}$.

By chain rule and $(2.8 \mathrm{~b})$

$$
\nabla \psi_{\varepsilon}(x, t)=\nabla_{q} d_{\varepsilon}^{F}\left(Q_{\varepsilon}\right): \nabla Q_{\varepsilon}(x, t) \quad \text { for a.e. }(x, t) \in \Omega \times(0, T) .
$$

This motivates the definition of the following projection of $\partial_{i} Q_{\varepsilon}$ onto the span of $\nabla_{q} d_{\varepsilon}^{F}\left(Q_{\varepsilon}\right)$

$$
\Pi_{Q_{\varepsilon}} \partial_{i} Q_{\varepsilon}= \begin{cases}\left(\partial_{i} Q_{\varepsilon}: \frac{\nabla_{q} d_{\varepsilon}^{F}\left(Q_{\varepsilon}\right)}{\left|\nabla_{q} d_{\varepsilon}^{F}\left(Q_{\varepsilon}\right)\right|}\right) \frac{\nabla_{q} d_{\varepsilon}^{F}\left(Q_{\varepsilon}\right)}{\left|\nabla_{q} d_{\varepsilon}^{F}\left(Q_{\varepsilon}\right)\right|}, & \text { if } \nabla_{q} d_{\varepsilon}^{F}\left(Q_{\varepsilon}\right) \neq 0, \\ 0, & \text { otherwise. }\end{cases}
$$

Hence, (4.9) implies

$$
\begin{gathered}
\left|\nabla \psi_{\varepsilon}\right|=\left|\Pi_{Q_{\varepsilon}} \nabla Q_{\varepsilon}\right|\left|\nabla_{q} d_{\varepsilon}^{F}\left(Q_{\varepsilon}\right)\right| \quad \text { for a.e. }(x, t) \in \Omega \times(0, T), \\
\Pi_{Q_{\varepsilon}} \nabla Q_{\varepsilon}=\frac{\left|\nabla \psi_{\varepsilon}\right|}{\left|\nabla_{q} d_{\varepsilon}^{F}\left(Q_{\varepsilon}\right)\right|^{2}} \nabla_{q} d_{\varepsilon}^{F}\left(Q_{\varepsilon}\right) \otimes \mathrm{n}_{\varepsilon} \quad \text { for a.e. }(x, t) \in \Omega \times(0, T),
\end{gathered}
$$

The next inequality will be crucial to show the non-negativity of the modulated energy (2.7) and various lower bounds of it. It states that the upper bound for the gradient of the convolution $d_{\varepsilon}^{F}$ is as good as if $d^{F}$ was $C^{1,1 / 2}$ and it simply follows from the fact that the modulus $\left|\nabla d^{F}\right|$ is $C^{1 / 2}$.

Lemma 4.1. For each $c_{0}>0$ there exists $\varepsilon_{0} \in \mathbb{R}^{+}$such that

$$
\left|\nabla_{q} d_{\varepsilon}^{F}(q)\right| \leqq \sqrt{2 F_{\varepsilon}(q)}, \quad \forall q \in \mathcal{Q},|q| \leqq c_{0}, \forall \varepsilon \in\left(0, \varepsilon_{0}\right) .
$$

Proof. Recall (2.8a), i.e. $F_{\varepsilon}(q)=F(q)+\varepsilon^{K-1}$ with $K=4$. It follows from (2.9), (3.1) and $\int_{\mathbb{R}^{5}} \phi_{\varepsilon}(p) \mathrm{d} p=1$ that

$$
\begin{aligned}
\left|\nabla_{q} d_{\varepsilon}^{F}(q)\right| & =\left|\int_{\mathbb{R}^{5}} \phi_{\varepsilon}(p) \nabla_{q} d^{F}(q-p) \mathrm{d} p\right| \\
& \leqq \int_{\mathbb{R}^{5}} \sqrt{\phi_{\varepsilon}(p)} \sqrt{\phi_{\varepsilon}(p)} \sqrt{2 F(q-p)} \mathrm{d} p \\
& \leqq \sqrt{\int_{\mathbb{R}^{5}} \phi_{\varepsilon}(p) 2 F(q-p) \mathrm{d} p} \\
& \leqq \sqrt{\int_{\mathbb{R}^{5}} \phi_{\varepsilon}(p)\left(2 F(q)+C_{0}|p|\right) \mathrm{d} p}
\end{aligned}
$$

where in the last step $C_{0}$ is a local Lipschitz constant of $F(q)$ for $|q| \leqq c_{0}$. By (2.9) and the assumption that $\phi$ is supported in the unit ball of $\mathcal{Q}$, the integral in the last step can be treated as follows

$$
\left|\nabla_{q} d_{\varepsilon}^{F}(q)\right| \leqq \sqrt{2 F(q)+C_{0} \varepsilon^{K} \int_{\mathbb{R}^{5}} \phi_{\varepsilon}(p)|p| \varepsilon^{-K} \mathrm{~d} p} \leqq \sqrt{2 F(q)+C_{0} \varepsilon^{K}}
$$

Finally choosing $\varepsilon_{0}$ sufficiently small leads to (4.12). 
We shall apply the above lemma with $c_{0}$ being the constant in (3.29).

As we shall not integrate the time variable $t$ throughout this section, we shall abbreviate the spatial integration $\int_{\Omega}$ by $\int$ and sometimes we omit the $\mathrm{d} x$. The following lemma shows that the energy $E_{\varepsilon}\left[Q_{\varepsilon} \mid I\right]$ defined by (2.7) controls various quantities:

Lemma 4.2. There exists a universal constant $C<\infty$ which is independent of $t \in(0, T)$ and $\varepsilon$ such that the following estimates hold for every $t \in(0, T)$ :

$$
\begin{aligned}
\int\left(\frac{\varepsilon}{2}\left|\nabla Q_{\varepsilon}\right|^{2}+\frac{1}{\varepsilon} F_{\varepsilon}\left(Q_{\varepsilon}\right)-\left|\nabla \psi_{\varepsilon}\right|\right) \mathrm{d} x & \leqq E_{\varepsilon}\left[Q_{\varepsilon} \mid I\right](t), \\
\frac{1}{2} \int\left(\sqrt{\varepsilon}\left|\Pi_{Q_{\varepsilon}} \nabla Q_{\varepsilon}\right|-\frac{1}{\sqrt{\varepsilon}} \sqrt{2 F_{\varepsilon}\left(Q_{\varepsilon}\right)}\right)^{2} \mathrm{~d} x & \\
+\frac{\varepsilon}{2} \int\left(\left|\nabla Q_{\varepsilon}-\Pi_{Q_{\varepsilon}} \nabla Q_{\varepsilon}\right|^{2}\right) \mathrm{d} x & \leqq E_{\varepsilon}\left[Q_{\varepsilon} \mid I\right](t), \\
\frac{1}{2} \int\left(\sqrt{\varepsilon}\left|\Pi_{Q_{\varepsilon}} \nabla Q_{\varepsilon}\right|-\frac{1}{\sqrt{\varepsilon}}\left|\nabla_{q} d_{\varepsilon}^{F}\left(Q_{\varepsilon}\right)\right|\right)^{2} \mathrm{~d} x & \leqq E_{\varepsilon}\left[Q_{\varepsilon} \mid I\right](t), \\
+\int\left(1-\xi \cdot \mathrm{n}_{\varepsilon}\right)\left(\frac{\varepsilon}{2}\left|\Pi_{Q_{\varepsilon}} \nabla Q_{\varepsilon}\right|^{2}+\left|\nabla \psi_{\varepsilon}\right|\right) \mathrm{d} x & \leqq C E_{\varepsilon}\left[Q_{\varepsilon} \mid I\right](t), \\
\left.\int\left(\frac{\varepsilon}{2}\left|\nabla Q_{\varepsilon}\right|^{2}+\frac{1}{\varepsilon} F_{\varepsilon}\left(Q_{\varepsilon}\right)+\left|\nabla \psi_{\varepsilon}\right|\right) \min \left(d^{2}\left(x, I_{t}\right), 1\right) \mathrm{d} x\right) & \left.\leqq C E_{\varepsilon}\left[Q_{\varepsilon} \mid I\right](t) .13 \mathrm{~d}\right)
\end{aligned}
$$

Proof of Lemma 4.2. Since $\left|\xi \cdot \nabla \psi_{\varepsilon}\right| \leqq\left|\nabla \psi_{\varepsilon}\right|$, we obtain the first estimate (4.13a). The second estimate ( $4.13 \mathrm{~b}$ ) follows from the first one by using the chain rule in form of (4.11a) for the term $\left|\nabla \psi_{\varepsilon}\right|$, the Lipschitz estimate (4.12) and then completing the square. Similarly, using the Lipschitz estimate (4.12) to the term $\frac{1}{\varepsilon} F_{\varepsilon}\left(Q_{\varepsilon}\right)$ instead yields (4.13c)

Let us now turn to the estimate (4.13d). Completing the square and using (4.12) yield

$$
\begin{aligned}
E_{\varepsilon}\left[Q_{\varepsilon} \mid I\right] \geqq & \frac{1}{2} \int\left(\sqrt{\varepsilon}\left|\nabla Q_{\varepsilon}\right|-\frac{1}{\sqrt{\varepsilon}}\left|\nabla_{q} d_{\varepsilon}^{F}\left(Q_{\varepsilon}\right)\right|\right)^{2} \mathrm{~d} x \\
& +\int\left(\left|\nabla_{q} d_{\varepsilon}^{F}\left(Q_{\varepsilon}\right)\right|\left|\nabla Q_{\varepsilon}\right|-\left|\nabla \psi_{\varepsilon}\right|\right) \mathrm{d} x \\
& +\int\left(1-\xi \cdot \mathrm{n}_{\varepsilon}\right)\left|\nabla \psi_{\varepsilon}\right| \mathrm{d} x
\end{aligned}
$$


By the chain rule in form of (4.11a), the second right-hand side integral is nonnegative. Using (4.11b) and Young's inequality, it holds that

$$
\begin{aligned}
\varepsilon\left|\Pi_{Q_{\varepsilon}} \nabla Q_{\varepsilon}\right|^{2} & =\left|\nabla \psi_{\varepsilon}\right|+\sqrt{\varepsilon}\left|\Pi_{Q_{\varepsilon}} \nabla Q_{\varepsilon}\right|\left(\sqrt{\varepsilon}\left|\Pi_{Q_{\varepsilon}} \nabla Q_{\varepsilon}\right|-\frac{\left|\nabla_{q} d_{\varepsilon}^{F}\left(Q_{\varepsilon}\right)\right|}{\sqrt{\varepsilon}}\right) \\
& \leqq\left|\nabla \psi_{\varepsilon}\right|+\frac{\varepsilon}{2}\left|\Pi_{Q_{\varepsilon}} \nabla Q_{\varepsilon}\right|^{2}+\frac{1}{2}\left(\sqrt{\varepsilon}\left|\Pi_{Q_{\varepsilon}} \nabla Q_{\varepsilon}\right|-\frac{\left|\nabla_{q} d_{\varepsilon}^{F}\left(Q_{\varepsilon}\right)\right|}{\sqrt{\varepsilon}}\right)^{2} .
\end{aligned}
$$

Hence

$$
\frac{\varepsilon}{2}\left|\Pi_{Q_{\varepsilon}} \nabla Q_{\varepsilon}\right|^{2} \leqq\left|\nabla \psi_{\varepsilon}\right|+\frac{1}{2}\left(\sqrt{\varepsilon}\left|\Pi_{Q_{\varepsilon}} \nabla Q_{\varepsilon}\right|-\frac{\left|\nabla_{q} d_{\varepsilon}^{F}\left(Q_{\varepsilon}\right)\right|}{\sqrt{\varepsilon}}\right)^{2} .
$$

This combined with (4.14), (4.13c) and the trivial estimate $1-\xi \cdot \mathrm{n}_{\varepsilon} \leqq 2$ leads to (4.13d). Finally, by $(2.6)$ and $\delta_{I} \in(0,1)$ we have

$$
1-\xi \cdot \mathrm{n}_{\varepsilon} \geqq 1-\eta \geqq \min \left(d^{2}\left(x, I_{t}\right), \frac{\delta_{I}^{2}}{4}\right) \geqq \frac{\delta_{I}^{2}}{4} \min \left(d^{2}\left(x, I_{t}\right), 1\right) .
$$

Applying this to the second right-hand side integral of (4.13d) and then using (4.13a) yield (4.13e).

The following result was first proved in [12] in the case of the Allen-Cahn equation, and can be generalized to the vectorial case:

Proposition 4.3. There exists a constant $C=C\left(I_{t}\right)$ depending on the interface $I_{t}$ such that

$$
\begin{aligned}
& \frac{\mathrm{d}}{\mathrm{d} t} E_{\varepsilon}\left[Q_{\varepsilon} \mid I\right]+\frac{1}{2 \varepsilon} \int\left(\varepsilon^{2}\left|\partial_{t} Q_{\varepsilon}\right|^{2}-\left|\mathrm{H}_{\varepsilon}\right|^{2}\right) \mathrm{d} x \\
& \quad+\frac{1}{2 \varepsilon} \int\left|\varepsilon \partial_{t} Q_{\varepsilon}-(\nabla \cdot \xi) \nabla_{q} d_{\varepsilon}^{F}\left(Q_{\varepsilon}\right)\right|^{2} \mathrm{~d} x \\
& \quad+\frac{1}{2 \varepsilon} \int\left|\mathrm{H}_{\varepsilon}-\varepsilon\right| \nabla Q_{\varepsilon}\left|\mathrm{H}_{I}\right|^{2} \mathrm{~d} x \leqq C E_{\varepsilon}\left[Q_{\varepsilon} \mid I\right] .
\end{aligned}
$$

The following lemma, the proof of which will be given at the end of this section, provides the exact computation of the time derivative of the energy $E_{\varepsilon}\left[Q_{\varepsilon} \mid I\right]$ : 
Lemma 4.4. Under the assumptions of Theorem 2.1, the following identity holds:

$$
\begin{aligned}
& \quad \frac{\mathrm{d}}{\mathrm{d} t} E\left[Q_{\varepsilon} \mid I\right]+\frac{1}{2 \varepsilon} \int\left|\varepsilon \partial_{t} Q_{\varepsilon}-(\nabla \cdot \xi) \nabla_{q} d_{\varepsilon}^{F}\left(Q_{\varepsilon}\right)\right|^{2} \mathrm{~d} x \\
& \quad+\frac{1}{2 \varepsilon} \int\left|\mathrm{H}_{\varepsilon}-\varepsilon\right| \nabla Q_{\varepsilon}\left|\mathrm{H}_{I}\right|^{2} \mathrm{~d} x \\
& =\frac{1}{2 \varepsilon} \int|(\nabla \cdot \xi)| \nabla_{q} d_{\varepsilon}^{F}\left(Q_{\varepsilon}\right)\left|\mathrm{n}_{\varepsilon}+\varepsilon\right| \Pi_{Q_{\varepsilon}} \nabla Q_{\varepsilon}\left|\mathrm{H}_{I}\right|^{2} \mathrm{~d} x \\
& \quad+\frac{\varepsilon}{2} \int\left|\mathrm{H}_{I}\right|^{2}\left(\left|\nabla Q_{\varepsilon}\right|^{2}-\left|\Pi_{Q_{\varepsilon}} \nabla Q_{\varepsilon}\right|^{2}\right) d x-\int \nabla \mathrm{H}_{I}:\left(\xi-\mathrm{n}_{\varepsilon}\right)^{\otimes 2}\left|\nabla \psi_{\varepsilon}\right| \mathrm{d} x \\
& \quad+\int\left(\nabla \cdot \mathrm{H}_{I}\right)\left(\frac{\varepsilon}{2}\left|\nabla Q_{\varepsilon}\right|^{2}+\frac{1}{\varepsilon} F_{\varepsilon}\left(Q_{\varepsilon}\right)-\left|\nabla \psi_{\varepsilon}\right|\right) \mathrm{d} x \\
& \quad+\int\left(\nabla \cdot \mathrm{H}_{I}\right)\left(1-\xi \cdot \mathrm{n}_{\varepsilon}\right)\left|\nabla \psi_{\varepsilon}\right| \mathrm{d} x+J_{\varepsilon}^{1}+J_{\varepsilon}^{2},
\end{aligned}
$$

where we use the notation

$$
\begin{aligned}
J_{\varepsilon}^{1}:= & \int \nabla \mathrm{H}_{I}: \mathrm{n}_{\varepsilon} \otimes \mathrm{n}_{\varepsilon}\left(\left|\nabla \psi_{\varepsilon}\right|-\varepsilon\left|\nabla Q_{\varepsilon}\right|^{2}\right) \mathrm{d} x \\
& +\varepsilon \int \nabla \mathrm{H}_{I}:\left(\mathrm{n}_{\varepsilon} \otimes \mathrm{n}_{\varepsilon}\right)\left(\left|\nabla Q_{\varepsilon}\right|^{2}-\left|\Pi_{Q_{\varepsilon}} \nabla Q_{\varepsilon}\right|^{2}\right) \mathrm{d} x \\
& -\varepsilon \int \sum_{i, j=1}^{3}\left(\nabla \mathrm{H}_{I}\right)_{i j}\left(\left(\partial_{i} Q_{\varepsilon}-\Pi_{Q_{\varepsilon}} \partial_{i} Q_{\varepsilon}\right):\left(\partial_{j} Q_{\varepsilon}-\Pi_{Q_{\varepsilon}} \partial_{j} Q_{\varepsilon}\right)\right) \mathrm{d} x,(4 \\
J_{\varepsilon}^{2}:= & -\int\left(\partial_{t} \xi+\left(\mathrm{H}_{I} \cdot \nabla\right) \xi+\left(\nabla \mathrm{H}_{I}\right)^{T} \xi\right) \cdot\left(\mathrm{n}_{\varepsilon}-\xi\right)\left|\nabla \psi_{\varepsilon}\right| \mathrm{d} x \\
& -\int\left(\partial_{t} \xi+\left(\mathrm{H}_{I} \cdot \nabla\right) \xi\right) \cdot \xi\left|\nabla \psi_{\varepsilon}\right| \mathrm{d} x
\end{aligned}
$$

In order to prove the proposition, we only need to estimate the terms on the RHS of (4.19).

Proof of Proposition 4.3. According to Lemma 4.4, we only need to estimate the RHS of (4.19) by $E_{\varepsilon}\left[Q_{\varepsilon} \mid I\right]$ up to a constant that only depends on $I_{t}$. We start with (4.19a): it follows from the triangle inequality that

$$
\begin{aligned}
\frac{1}{2} \int \mid & \frac{1}{\sqrt{\varepsilon}}(\nabla \cdot \xi)\left|\nabla_{q} d_{\varepsilon}^{F}\left(Q_{\varepsilon}\right)\right| \mathrm{n}_{\varepsilon}+\left.\sqrt{\varepsilon}\left|\Pi_{Q_{\varepsilon}} \nabla Q_{\varepsilon}\right| \mathrm{H}_{I}\right|^{2} \mathrm{~d} x \\
\leqq & \left|(\nabla \cdot \xi)\left(\sqrt{\varepsilon}\left|\Pi_{Q_{\varepsilon}} \nabla Q_{\varepsilon}\right|-\frac{1}{\sqrt{\varepsilon}}\left|\nabla_{q} d_{\varepsilon}^{F}\left(Q_{\varepsilon}\right)\right|\right) \mathrm{n}_{\varepsilon}\right|^{2} \mathrm{~d} x \\
& +\int|(\nabla \cdot \xi) \sqrt{\varepsilon}| \Pi_{Q_{\varepsilon}} \nabla Q_{\varepsilon}\left|\left(\mathrm{n}_{\varepsilon}-\xi\right)\right|^{2} \mathrm{~d} x \\
& +\int\left|\left(\mathrm{H}_{I}+(\nabla \cdot \xi) \xi\right) \sqrt{\varepsilon}\right| \Pi_{Q_{\varepsilon}} \nabla Q_{\varepsilon}||^{2} \mathrm{~d} x .
\end{aligned}
$$


The first integral is controlled by (4.13c). Using (4.4), the second integral is controlled by $(4.13 \mathrm{~d})$. The third integral can be treated using the relation $\mathrm{H}_{I}=$ $\left(\mathrm{H}_{I} \cdot \xi\right) \xi+O\left(d\left(x, I_{t}\right)\right)$ and (4.4), so it can be controlled by (4.13e).

The integrals in (4.19b) can be controlled using (4.13b) and (4.13d), recalling that

$$
\left|\xi-\mathrm{n}_{\varepsilon}\right|^{2} \leqq 2\left(1-\mathrm{n}_{\varepsilon} \cdot \xi\right)
$$

The first term in (4.19c) can be controlled using (4.13a), and the second term can be estimated by (4.13d). It remains to estimate (4.20) and (4.21). The last two terms on the RHS of $J_{\varepsilon}^{1}$ can be bounded using (4.13b), and the first integral can be rewritten using $\mathrm{n}_{\varepsilon}=\mathrm{n}_{\varepsilon}-\xi+\xi$ :

$$
\begin{aligned}
J_{\varepsilon}^{1} \leqq & \int \nabla \mathrm{H}_{I}:\left(\mathrm{n}_{\varepsilon} \otimes\left(\mathrm{n}_{\varepsilon}-\xi\right)\right)\left(\left|\nabla \psi_{\varepsilon}\right|-\varepsilon\left|\nabla Q_{\varepsilon}\right|^{2}\right) \mathrm{d} x \\
& +\int \nabla \mathrm{H}_{I}: \mathrm{n}_{\varepsilon} \otimes \xi\left(\left|\nabla \psi_{\varepsilon}\right|-\varepsilon\left|\nabla Q_{\varepsilon}\right|^{2}\right) \mathrm{d} x+C E_{\varepsilon}\left[Q_{\varepsilon} \mid I\right] \\
\leqq & \left\|\nabla \mathrm{H}_{I}\right\|_{L^{\infty}} \int\left|\mathrm{n}_{\varepsilon}-\xi\right|\left(\varepsilon\left|\nabla Q_{\varepsilon}\right|^{2}-\varepsilon\left|\Pi_{Q_{\varepsilon}} \nabla Q_{\varepsilon}\right|^{2}\right. \\
& \left.+\left.|\varepsilon| \Pi_{Q_{\varepsilon}} \nabla Q_{\varepsilon}\right|^{2}-\left|\nabla \psi_{\varepsilon}\right| \mid\right) \mathrm{d} x \\
& +C \int \min \left(d^{2}\left(x, I_{t}\right), 1\right)\left(\left|\nabla \psi_{\varepsilon}\right|+\varepsilon\left|\nabla Q_{\varepsilon}\right|^{2}\right) \mathrm{d} x+C E_{\varepsilon}\left[Q_{\varepsilon} \mid I\right] .
\end{aligned}
$$

Note that in the last step we employed

$$
\nabla \mathrm{H}_{I}: \mathrm{n}_{\varepsilon} \otimes \xi=\left(\xi \cdot \nabla \mathrm{H}_{I}\right) \cdot \mathrm{n}_{\varepsilon}
$$

and the fact that $(\xi \cdot \nabla) \mathrm{H}_{I}$ vanishes in the neighborhood $I_{t}\left(\frac{\delta_{I}}{2}\right)$ by definition (4.2). Thus we employ (4.13d) and (4.13b), and (4.11a) to get

$$
\begin{aligned}
J_{\varepsilon}^{1} \leqq & \left.C \int\left|\mathrm{n}_{\varepsilon}-\xi\right||\varepsilon| \Pi_{Q_{\varepsilon}} \nabla Q_{\varepsilon}\right|^{2}-\left|\nabla \psi_{\varepsilon}\right| \mid \mathrm{d} x+C E_{\varepsilon}\left[Q_{\varepsilon} \mid I\right] \\
= & C \int\left|\mathrm{n}_{\varepsilon}-\xi\right| \sqrt{\varepsilon}\left|\Pi_{Q_{\varepsilon}} \nabla Q_{\varepsilon}\right||\sqrt{\varepsilon}| \Pi_{Q_{\varepsilon}} \nabla Q_{\varepsilon}\left|-\frac{\left|\nabla_{q} d_{\varepsilon}^{F}\left(Q_{\varepsilon}\right)\right|}{\sqrt{\varepsilon}}\right| \mathrm{d} x \\
& +C E_{\varepsilon}\left[Q_{\varepsilon} \mid I\right] .
\end{aligned}
$$

Finally applying the Cauchy-Schwarz inequality and then (4.13d) and (4.13b), we obtain

$$
J_{\varepsilon}^{1} \leqq C E_{\varepsilon}\left[Q_{\varepsilon} \mid I\right]
$$

As for $J_{\varepsilon}^{2}$ (4.21), we employ (4.3a) and (4.3b) and yield

$$
J_{\varepsilon}^{2} \leqq C \int\left(\left|\mathrm{n}_{\varepsilon}-\xi\right|^{2}+\min \left(d^{2}\left(x, I_{t}\right), 1\right)\right)\left|\nabla \psi_{\varepsilon}\right| \mathrm{d} x \leqq C E_{\varepsilon}\left[Q_{\varepsilon} \mid I\right],
$$

after applying (4.13d) and (4.13e). Therefore we have proved that the RHS of (4.19) is bounded by $E_{\varepsilon}\left[Q_{\varepsilon} \mid I\right]$ up to a multiplicative constant which only depends on $I_{t}$. 
The following lemma will be used in the proof of Lemma 4.4:

Lemma 4.5. Under the assumptions of Theorem 2.1,

$$
\begin{aligned}
& \int \mathrm{H}_{I}:\left(\xi \otimes \mathrm{n}_{\varepsilon}\right)\left|\nabla \psi_{\varepsilon}\right| \mathrm{d} x-\int\left(\nabla \cdot \mathrm{H}_{I}\right) \xi \cdot \nabla \psi_{\varepsilon} \mathrm{d} x \\
& =\int \nabla \mathrm{H}_{I}:\left(\xi-\mathrm{n}_{\varepsilon}\right) \otimes \mathrm{n}_{\varepsilon}\left|\nabla \psi_{\varepsilon}\right| \mathrm{d} x+\int \mathrm{H}_{\varepsilon} \cdot \mathrm{H}_{I}\left|\nabla Q_{\varepsilon}\right| \mathrm{d} x \\
& \quad+\int \nabla \cdot \mathrm{H}_{I}\left(\frac{\varepsilon}{2}\left|\nabla Q_{\varepsilon}\right|^{2}+\frac{1}{\varepsilon} F_{\varepsilon}\left(Q_{\varepsilon}\right)-\left|\nabla \psi_{\varepsilon}\right|\right) \mathrm{d} x \\
& \quad+\int \nabla \cdot \mathrm{H}_{I}\left(\left|\nabla \psi_{\varepsilon}\right|-\xi \cdot \nabla \psi_{\varepsilon}\right) \mathrm{d} x \\
& \quad-\int \sum_{i, j=1}^{3}\left(\nabla \mathrm{H}_{I}\right)_{i j} \varepsilon\left(\partial_{i} Q_{\varepsilon}: \partial_{j} Q_{\varepsilon}\right) \mathrm{d} x+\int \nabla \mathrm{H}_{I}:\left(\mathrm{n}_{\varepsilon} \otimes \mathrm{n}_{\varepsilon}\right)\left|\nabla \psi_{\varepsilon}\right| \mathrm{d} x
\end{aligned}
$$

Proof. The LHS of (4.26) can be written as

$$
\begin{aligned}
& \int \nabla \mathrm{H}_{I}:\left(\xi \otimes \mathrm{n}_{\varepsilon}\right)\left|\nabla \psi_{\varepsilon}\right| \mathrm{d} x-\int\left(\nabla \cdot \mathrm{H}_{I}\right) \xi \cdot \nabla \psi_{\varepsilon} \mathrm{d} x \\
& =\int \nabla \mathrm{H}_{I}:\left(\xi-\mathrm{n}_{\varepsilon}\right) \otimes \mathrm{n}_{\varepsilon}\left|\nabla \psi_{\varepsilon}\right| \mathrm{d} x+\int \nabla \mathrm{H}_{I}:\left(\mathrm{n}_{\varepsilon} \otimes \mathrm{n}_{\varepsilon}\right)\left|\nabla \psi_{\varepsilon}\right| \mathrm{d} x \\
& \quad-\int\left(\nabla \cdot \mathrm{H}_{I}\right) \xi \cdot \nabla \psi_{\varepsilon} \mathrm{d} x .
\end{aligned}
$$

To treat the second term on the RHS of (4.26), we introduce the energy stress tensor $T_{\varepsilon}$

$$
\left(T_{\varepsilon}\right)_{i j}=\left(\frac{\varepsilon}{2}\left|\nabla Q_{\varepsilon}\right|^{2}+\frac{1}{\varepsilon} F_{\varepsilon}\left(Q_{\varepsilon}\right)\right) \delta_{i j}-\varepsilon \partial_{i} Q_{\varepsilon}: \partial_{j} Q_{\varepsilon}
$$

In view of (4.8a), we have the identity

$$
\nabla \cdot T_{\varepsilon}=-\varepsilon \nabla Q_{\varepsilon}: \Delta Q_{\varepsilon}+\frac{1}{\varepsilon} \nabla_{q} F_{\varepsilon}\left(Q_{\varepsilon}\right): \nabla Q_{\varepsilon}=\mathrm{H}_{\varepsilon}\left|\nabla Q_{\varepsilon}\right|
$$

Testing this identity by $\mathrm{H}_{I}$, integrating by parts and using (4.6), we obtain

$$
\begin{aligned}
\int \mathrm{H}_{\varepsilon} \cdot \mathrm{H}_{I}\left|\nabla Q_{\varepsilon}\right| \mathrm{d} x= & -\int \nabla \mathrm{H}_{I}: T_{\varepsilon} \mathrm{d} x \\
= & -\int \nabla \cdot \mathrm{H}_{I}\left(\frac{\varepsilon}{2}\left|\nabla Q_{\varepsilon}\right|^{2}+\frac{1}{\varepsilon} F_{\varepsilon}\left(Q_{\varepsilon}\right)\right) \mathrm{d} x \\
& +\int \sum_{i, j=1}^{3}\left(\nabla \mathrm{H}_{I}\right)_{i j} \varepsilon\left(\partial_{i} Q_{\varepsilon}: \partial_{j} Q_{\varepsilon}\right) \mathrm{d} x
\end{aligned}
$$


Adding zero leads to

$$
\begin{aligned}
\int & \nabla \mathrm{H}_{I}: \mathrm{n}_{\varepsilon} \otimes \mathrm{n}_{\varepsilon}\left|\nabla \psi_{\varepsilon}\right| \mathrm{d} x \\
= & \int \mathrm{H}_{\varepsilon} \cdot \mathrm{H}_{I}\left|\nabla Q_{\varepsilon}\right| \mathrm{d} x+\int \nabla \cdot \mathrm{H}_{I}\left(\frac{\varepsilon}{2}\left|\nabla Q_{\varepsilon}\right|^{2}+\frac{1}{\varepsilon} F_{\varepsilon}\left(Q_{\varepsilon}\right)-\left|\nabla \psi_{\varepsilon}\right|\right) \mathrm{d} x \\
& +\int \nabla \cdot \mathrm{H}_{I}\left|\nabla \psi_{\varepsilon}\right| \mathrm{d} x \\
& -\int \sum_{i, j=1}^{3}\left(\nabla \mathrm{H}_{I}\right)_{i j} \varepsilon\left(\partial_{i} Q_{\varepsilon}: \partial_{j} Q_{\varepsilon}\right) \mathrm{d} x+\int\left(\nabla \mathrm{H}_{I}\right):\left(\mathrm{n}_{\varepsilon} \otimes \mathrm{n}_{\varepsilon}\right)\left|\nabla \psi_{\varepsilon}\right| \mathrm{d} x .
\end{aligned}
$$

Substituting this identity into (4.27) leads to (4.26).

Proof of Lemma 4.4. Using the energy dissipation law (4.1) and adding zero, we compute the time derivative of the energy (2.7) by

$$
\begin{aligned}
& \frac{\mathrm{d}}{\mathrm{d} t} E_{\varepsilon}\left[Q_{\varepsilon} \mid I\right]+\varepsilon \int\left|\partial_{t} Q_{\varepsilon}\right|^{2} \mathrm{~d} x-\int(\nabla \cdot \xi) \nabla_{q} d_{\varepsilon}^{F}\left(Q_{\varepsilon}\right): \partial_{t} Q_{\varepsilon} \mathrm{d} x \\
& =\int\left(\mathrm{H}_{I} \cdot \nabla\right) \xi \cdot \nabla \psi_{\varepsilon} \mathrm{d} x+\int\left(\nabla \mathrm{H}_{I}\right)^{T} \xi \cdot \nabla \psi_{\varepsilon} \mathrm{d} x \\
& \quad-\int\left(\partial_{t} \xi+\left(\mathrm{H}_{I} \cdot \nabla\right) \xi+\left(\nabla \mathrm{H}_{I}\right)^{T} \xi\right) \cdot \nabla \psi_{\varepsilon} \mathrm{d} x .
\end{aligned}
$$

Due to the symmetry of the Hessian of $\psi_{\varepsilon}$ and the boundary conditions (4.6), we have

$$
\int \nabla \cdot\left(\xi \otimes \mathrm{H}_{I}\right) \cdot \nabla \psi_{\varepsilon} \mathrm{d} x=\int \nabla \cdot\left(\mathrm{H}_{I} \otimes \xi\right) \cdot \nabla \psi_{\varepsilon} \mathrm{d} x .
$$

Hence, the first integral on the RHS above can be rewritten as

$$
\begin{aligned}
\int\left(\mathrm{H}_{I} \cdot \nabla\right) \xi \cdot \nabla \psi_{\varepsilon} \mathrm{d} x= & \int \nabla \cdot\left(\xi \otimes \mathrm{H}_{I}\right) \cdot \nabla \psi_{\varepsilon} \mathrm{d} x-\int\left(\nabla \cdot \mathrm{H}_{I}\right) \xi \cdot \nabla \psi_{\varepsilon} \mathrm{d} x \\
= & \int(\nabla \cdot \xi) \mathrm{H}_{I} \cdot \nabla \psi_{\varepsilon} \mathrm{d} x+\int(\xi \cdot \nabla) \mathrm{H}_{I} \cdot \nabla \psi_{\varepsilon} \mathrm{d} x \\
& -\int\left(\nabla \cdot \mathrm{H}_{I}\right) \xi \cdot \nabla \psi_{\varepsilon} \mathrm{d} x .
\end{aligned}
$$

Therefore

$$
\begin{aligned}
& \frac{\mathrm{d}}{\mathrm{d} t} E_{\varepsilon}\left[Q_{\varepsilon} \mid I\right]+\varepsilon \int\left|\partial_{t} Q_{\varepsilon}\right|^{2} \mathrm{~d} x-\int(\nabla \cdot \xi) \nabla_{q} d_{\varepsilon}^{F}\left(Q_{\varepsilon}\right): \partial_{t} Q_{\varepsilon} \mathrm{d} x \\
= & \int(\nabla \cdot \xi) \mathrm{H}_{I} \cdot \nabla \psi_{\varepsilon} \mathrm{d} x+\int(\xi \cdot \nabla) \mathrm{H}_{I} \cdot \nabla \psi_{\varepsilon} \mathrm{d} x-\int\left(\nabla \cdot \mathrm{H}_{I}\right) \xi \cdot \nabla \psi_{\varepsilon} \mathrm{d} x \\
& +\int \nabla \mathrm{H}_{I}:\left(\xi \otimes \mathrm{n}_{\varepsilon}\right)\left|\nabla \psi_{\varepsilon}\right| \mathrm{d} x-\int\left(\partial_{t} \xi+\left(\mathrm{H}_{I} \cdot \nabla\right) \xi+\left(\nabla \mathrm{H}_{I}\right)^{T} \xi\right) \cdot \nabla \psi_{\varepsilon} \mathrm{d} x .
\end{aligned}
$$


Using (4.26) in Lemma 4.5 to replace the third and fourth integrals on the RHS of the above identity and rewriting the last integral, we arrive at

$$
\begin{aligned}
\frac{\mathrm{d}}{\mathrm{d} t} E_{\varepsilon}\left[Q_{\varepsilon} \mid I\right]+\varepsilon \int\left|\partial_{t} Q_{\varepsilon}\right|^{2} \mathrm{~d} x-\int(\nabla \cdot \xi) \nabla_{q} d_{\varepsilon}^{F}\left(Q_{\varepsilon}\right): \partial_{t} Q_{\varepsilon} \mathrm{d} x \\
=\int(\nabla \cdot \xi) \mathrm{H}_{I} \cdot \nabla \psi_{\varepsilon} \mathrm{d} x+\int(\xi \cdot \nabla) \mathrm{H}_{I} \cdot \nabla \psi_{\varepsilon} \mathrm{d} x \\
+\int \nabla \mathrm{H}_{I}:\left(\xi-\mathrm{n}_{\varepsilon}\right) \otimes \mathrm{n}_{\varepsilon}\left|\nabla \psi_{\varepsilon}\right| \mathrm{d} x \\
+\int \mathrm{H}_{\varepsilon} \cdot \mathrm{H}_{I}\left|\nabla Q_{\varepsilon}\right| \mathrm{d} x+\int \nabla \cdot \mathrm{H}_{I}\left(\frac{\varepsilon}{2}\left|\nabla Q_{\varepsilon}\right|^{2}+\frac{1}{\varepsilon} F_{\varepsilon}\left(Q_{\varepsilon}\right)-\left|\nabla \psi_{\varepsilon}\right|\right) \mathrm{d} x \\
+\int \nabla \cdot \mathrm{H}_{I}\left(\left|\nabla \psi_{\varepsilon}\right|-\xi \cdot \nabla \psi_{\varepsilon}\right) \mathrm{d} x \\
\quad-\int \sum_{i, j=1}^{3}\left(\nabla \mathrm{H}_{I}\right)_{i j} \varepsilon\left(\partial_{i} Q_{\varepsilon}: \partial_{j} Q_{\varepsilon}\right) \mathrm{d} x+\int \nabla \mathrm{H}_{I}: \mathrm{n}_{\varepsilon} \otimes \mathrm{n}_{\varepsilon}\left|\nabla \psi_{\varepsilon}\right| \mathrm{d} x \\
\quad-\int\left(\partial_{t} \xi+\left(\mathrm{H}_{I} \cdot \nabla\right) \xi+\left(\nabla \mathrm{H}_{I}\right)^{T} \xi\right) \cdot\left(\mathrm{n}_{\varepsilon}-\xi\right)\left|\nabla \psi_{\varepsilon}\right| \mathrm{d} x \\
\quad-\int\left(\partial_{t} \xi+\left(\mathrm{H}_{I} \cdot \nabla\right) \xi\right) \cdot \xi\left|\nabla \psi_{\varepsilon}\right| \mathrm{d} x-\int\left(\nabla \mathrm{H}_{I}\right)^{T}:(\xi \otimes \xi)\left|\nabla \psi_{\varepsilon}\right| \mathrm{d} x
\end{aligned}
$$

First, note that the third to last and second to last integrals combine to $J_{\varepsilon}^{2}$. Next, by the property (4.11b) of the orthogonal projection (4.10), we can also find $J_{\varepsilon}^{1}$ on the right-hand side. Indeed,

$$
\begin{aligned}
& -\int \sum_{i, j=1}^{3}\left(\nabla \mathrm{H}_{I}\right)_{i j} \varepsilon\left(\partial_{i} Q_{\varepsilon}: \partial_{j} Q_{\varepsilon}\right) \mathrm{d} x+\int \nabla \mathrm{H}_{I}: \mathrm{n}_{\varepsilon} \otimes \mathrm{n}_{\varepsilon}\left|\nabla \psi_{\varepsilon}\right| \mathrm{d} x \\
& =\int \nabla \mathrm{H}_{I}: \mathrm{n}_{\varepsilon} \otimes \mathrm{n}_{\varepsilon}\left|\nabla \psi_{\varepsilon}\right| \mathrm{d} x-\varepsilon \int\left(\nabla \mathrm{H}_{I}\right)_{i j}\left(\Pi_{Q_{\varepsilon}} \partial_{i} Q_{\varepsilon}: \Pi_{Q_{\varepsilon}} \partial_{j} Q_{\varepsilon}\right) \mathrm{d} x \\
& \quad-\int \sum_{i, j=1}^{3}\left(\nabla \mathrm{H}_{I}\right)_{i j} \varepsilon\left(\left(\partial_{i} Q_{\varepsilon}-\Pi_{Q_{\varepsilon}} \partial_{i} Q_{\varepsilon}\right):\left(\partial_{j} Q_{\varepsilon}-\Pi_{Q_{\varepsilon}} \partial_{j} Q_{\varepsilon}\right)\right) \mathrm{d} x \\
& =\int \nabla \mathrm{H}_{I}: \mathrm{n}_{\varepsilon} \otimes \mathrm{n}_{\varepsilon}\left(\left|\nabla \psi_{\varepsilon}\right|-\varepsilon\left|\nabla Q_{\varepsilon}\right|^{2}\right) \mathrm{d} x \\
& \quad+\varepsilon \int \nabla \mathrm{H}_{I}:\left(\mathrm{n}_{\varepsilon} \otimes \mathrm{n}_{\varepsilon}\right)\left(\left|\nabla Q_{\varepsilon}\right|^{2}-\left|\Pi_{Q_{\varepsilon}} \nabla Q_{\varepsilon}\right|^{2}\right) \mathrm{d} x \\
& \quad-\int \sum_{i, j=1}^{3}\left(\nabla \mathrm{H}_{I}\right)_{i j} \varepsilon\left(\left(\partial_{i} Q_{\varepsilon}-\Pi_{Q_{\varepsilon}} \partial_{i} Q_{\varepsilon}\right):\left(\partial_{j} Q_{\varepsilon}-\Pi_{Q_{\varepsilon}} \partial_{j} Q_{\varepsilon}\right)\right) \mathrm{d} x=J_{\varepsilon}^{1} .
\end{aligned}
$$


Using the definition (4.8b) of $\mathrm{n}_{\varepsilon}$, we may merge the second, third, and the last integral on the RHS of (4.32) to obtain

$$
\begin{aligned}
\frac{\mathrm{d}}{\mathrm{d} t} E_{\varepsilon}\left[Q_{\varepsilon} \mid I\right]= & -\varepsilon \int\left|\partial_{t} Q_{\varepsilon}\right|^{2} \mathrm{~d} x+\int(\nabla \cdot \xi) \nabla_{q} d_{\varepsilon}^{F}\left(Q_{\varepsilon}\right): \partial_{t} Q_{\varepsilon} \mathrm{d} x \\
& +\int(\nabla \cdot \xi) \mathrm{H}_{I} \cdot \nabla \psi_{\varepsilon} \mathrm{d} x+\int \mathrm{H}_{\varepsilon} \cdot \mathrm{H}_{I}\left|\nabla Q_{\varepsilon}\right| \mathrm{d} x \\
& -\int \nabla \mathrm{H}_{I}:\left(\xi-\mathrm{n}_{\varepsilon}\right)^{\otimes 2}\left|\nabla \psi_{\varepsilon}\right| \mathrm{d} x \\
& +J_{\varepsilon}^{1}+\int\left(\nabla \cdot \mathrm{H}_{I}\right)\left(\frac{\varepsilon}{2}\left|\nabla Q_{\varepsilon}\right|^{2}+\frac{1}{\varepsilon} F_{\varepsilon}\left(Q_{\varepsilon}\right)-\left|\nabla \psi_{\varepsilon}\right|\right) \mathrm{d} x \\
& +\int\left(\nabla \cdot \mathrm{H}_{I}\right)\left(1-\xi \cdot \mathrm{n}_{\varepsilon}\right)\left|\nabla \psi_{\varepsilon}\right| \mathrm{d} x+J_{\varepsilon}^{2} .
\end{aligned}
$$

Now we complete squares for the first four terms on the RHS of (4.33): Reordering terms, we have

$$
\begin{aligned}
-\varepsilon \mid & \left.\partial_{t} Q_{\varepsilon}\right|^{2}+(\nabla \cdot \xi) \nabla_{q} d_{\varepsilon}^{F}\left(Q_{\varepsilon}\right): \partial_{t} Q_{\varepsilon}+(\nabla \cdot \xi) \mathrm{H}_{I} \cdot \nabla \psi_{\varepsilon}+\mathrm{H}_{\varepsilon} \cdot \mathrm{H}_{I}\left|\nabla Q_{\varepsilon}\right| \\
= & -\frac{1}{2 \varepsilon}\left(\left|\varepsilon \partial_{t} Q_{\varepsilon}\right|^{2}-2(\nabla \cdot \xi) \nabla_{q} d_{\varepsilon}^{F}\left(Q_{\varepsilon}\right): \varepsilon \partial_{t} Q_{\varepsilon}+(\nabla \cdot \xi)^{2}\left|\nabla_{q} d_{\varepsilon}^{F}\left(Q_{\varepsilon}\right)\right|^{2}\right) \\
& -\frac{1}{2 \varepsilon}\left|\varepsilon \partial_{t} Q_{\varepsilon}\right|^{2}+\frac{1}{2 \varepsilon}(\nabla \cdot \xi)^{2}\left|\nabla_{q} d_{\varepsilon}^{F}\left(Q_{\varepsilon}\right)\right|^{2}+(\nabla \cdot \xi) \mathrm{H}_{I} \cdot \nabla \psi_{\varepsilon} \\
& -\frac{1}{2 \varepsilon}\left(\left|\mathrm{H}_{\varepsilon}\right|^{2}-2 \mathrm{H}_{\varepsilon} \cdot \varepsilon\left|\nabla Q_{\varepsilon}\right| \mathrm{H}_{I}+\varepsilon^{2}\left|\nabla Q_{\varepsilon}\right|^{2}\left|\mathrm{H}_{I}\right|^{2}\right) \\
& +\frac{1}{2 \varepsilon}\left(\left|\mathrm{H}_{\varepsilon}\right|^{2}+\varepsilon^{2}\left|\nabla Q_{\varepsilon}\right|^{2}\left|\mathrm{H}_{I}\right|^{2}\right) \\
= & -\frac{1}{2 \varepsilon}\left|\varepsilon \partial_{t} Q_{\varepsilon}-(\nabla \cdot \xi) \nabla_{q} d_{\varepsilon}^{F}\left(Q_{\varepsilon}\right)^{2}-\frac{1}{2 \varepsilon}\right| \mathrm{H}_{\varepsilon}-\left.\varepsilon\left|\nabla Q_{\varepsilon}\right| \mathrm{H}_{I}\right|^{2} \\
& -\frac{1}{2 \varepsilon}\left|\varepsilon \partial_{t} Q_{\varepsilon}\right|^{2}+\frac{1}{2 \varepsilon}\left|\mathrm{H}_{\varepsilon}\right|^{2} \\
& +\frac{1}{2 \varepsilon}\left((\nabla \cdot \xi)^{2}\left|\nabla_{q} d_{\varepsilon}^{F}\left(Q_{\varepsilon}\right)\right|^{2}+2 \varepsilon(\nabla \cdot \xi) \nabla \psi_{\varepsilon} \cdot \mathrm{H}_{I}+\left|\varepsilon \Pi_{Q_{\varepsilon}} \nabla Q_{\varepsilon}\right|^{2}\left|\mathrm{H}_{I}\right|^{2}\right) \\
& +\frac{\varepsilon}{2}\left(\left|\nabla Q_{\varepsilon}\right|^{2}-\left|\Pi_{Q_{\varepsilon}} \nabla Q_{\varepsilon}\right|^{2}\right)\left|\mathrm{H}_{I}\right|^{2} .
\end{aligned}
$$

Using the definition (4.8b) of the normal $\mathrm{n}_{\varepsilon}$ and the chain rule in form of (4.11a), the terms in (4.33) form the last missing square. Integrating over the domain $\Omega$ and substituting into (4.33) we arrive at (4.19).

\section{Convergence to the Harmonic Map Heat Flow}

This section is devoted to the proof of Theorem 2.1. We start with a lemma about uniform estimates of $Q_{\varepsilon}$. 
Lemma 5.1. There exists a universal constant $C=C\left(I_{0}\right)$ such that

$$
\begin{aligned}
& \operatorname{ess}_{\sup _{t \in[0, T]}} \int_{\Omega}\left(\left|\nabla Q_{\varepsilon}-\Pi_{Q_{\varepsilon}} \nabla Q_{\varepsilon}\right|^{2}\right) \mathrm{d} x \\
& +\int_{0}^{T} \int_{\Omega}\left(\left|\partial_{t} Q_{\varepsilon}-\Pi_{Q_{\varepsilon}} \partial_{t} Q_{\varepsilon}\right|^{2}\right) \mathrm{d} x \mathrm{~d} t \leqq e^{(1+T) C\left(I_{0}\right)} .
\end{aligned}
$$

Moreover, for any fixed $\delta \in\left(0, \delta_{I}\right)$, if holds that

$$
\begin{aligned}
\operatorname{ess}_{\sup } \sin _{t \in T]} \int_{\Omega^{ \pm}(t) \backslash I_{t}(\delta)}\left(\left|\nabla Q_{\varepsilon}\right|^{2}+\frac{F\left(Q_{\varepsilon}\right)+\varepsilon^{3}}{\varepsilon^{2}}\right) \mathrm{d} x \leqq \delta^{-2} e^{(1+T) C\left(I_{0}\right)}, \\
\int_{0}^{T} \int_{\Omega^{ \pm}(t) \backslash I_{t}(\delta)}\left|\partial_{t} Q_{\varepsilon}\right|^{2} \mathrm{~d} x \mathrm{~d} t \leqq \delta^{-2} e^{(1+T) C\left(I_{0}\right)} .
\end{aligned}
$$

Proof. We first establish a priori estimates of the solutions $Q_{\varepsilon}$ which are independent of $\varepsilon$. It follows from (4.18) and the assumption (2.12) that

$$
\begin{aligned}
& \operatorname{ess}_{\sup _{t \in[0, T]}} \frac{1}{\varepsilon} E_{\varepsilon}\left[Q_{\varepsilon} \mid I\right](t)+\frac{1}{\varepsilon^{2}} \int_{0}^{T} \int_{\Omega}\left|\varepsilon \partial_{t} Q_{\varepsilon}-\nabla_{q} d_{\varepsilon}^{F}\left(Q_{\varepsilon}\right)(\nabla \cdot \xi)\right|^{2} \mathrm{~d} x \mathrm{~d} t \\
& +\frac{1}{\varepsilon^{2}} \int_{0}^{T} \int_{\Omega}\left(\varepsilon^{2}\left|\partial_{t} Q_{\varepsilon}\right|^{2}-\left|\mathrm{H}_{\varepsilon}\right|^{2}+\left|\mathrm{H}_{\varepsilon}-\varepsilon \mathrm{H}_{I}\right| \nabla Q_{\varepsilon}||^{2}\right) \mathrm{d} x \mathrm{~d} t \\
& \leqq \frac{e^{(1+T) C\left(I_{0}\right)}}{\varepsilon} E_{\varepsilon}\left[Q_{\varepsilon} \mid I\right](0) \leqq e^{(1+T) C\left(I_{0}\right)} .
\end{aligned}
$$

On the other hand, using the orthogonal projection (4.10), we obtain

$$
\begin{aligned}
\left|\varepsilon \partial_{t} Q_{\varepsilon}-\nabla_{q} d_{\varepsilon}^{F}\left(Q_{\varepsilon}\right)(\nabla \cdot \xi)\right|^{2}= & \left|\varepsilon \partial_{t} Q_{\varepsilon}-\varepsilon \Pi_{Q_{\varepsilon}} \partial_{t} Q_{\varepsilon}\right|^{2} \\
& +\left|\varepsilon \Pi_{Q_{\varepsilon}} \partial_{t} Q_{\varepsilon}-\nabla_{q} d_{\varepsilon}^{F}\left(Q_{\varepsilon}\right)(\nabla \cdot \xi)\right|^{2} .
\end{aligned}
$$

This, together with (5.4), yields

$$
\begin{aligned}
& \frac{1}{\varepsilon^{2}} \int_{0}^{T} \int_{\Omega}\left|\varepsilon \partial_{t} Q_{\varepsilon}-\varepsilon \Pi_{Q_{\varepsilon}} \partial_{t} Q_{\varepsilon}\right|^{2} \\
& \quad+\frac{1}{\varepsilon^{2}} \int_{0}^{T} \int_{\Omega}\left|\varepsilon \Pi_{Q_{\varepsilon}} \partial_{t} Q_{\varepsilon}-\nabla_{q} d_{\varepsilon}^{F}\left(Q_{\varepsilon}\right)(\nabla \cdot \xi)\right|^{2} \leqq e^{(1+T) C\left(I_{0}\right)}
\end{aligned}
$$

The above two estimates together with (4.13b) implies (5.1). Moreover, (5.2) follows from (5.4) and (4.13e). Now we turn to the time derivative. It follows from (5.4) that

$$
\frac{1}{\varepsilon^{2}} \int_{0}^{T} \int_{\Omega}\left(\varepsilon^{2}\left|\partial_{t} Q_{\varepsilon}\right|^{2}-\left|\mathrm{H}_{\varepsilon}\right|^{2}+\left|\mathrm{H}_{\varepsilon}-\varepsilon \mathrm{H}_{I}\right| \nabla Q_{\varepsilon}||^{2}\right) \mathrm{d} x \mathrm{~d} t \leqq e^{(1+T) C\left(I_{0}\right)}
$$


By (1.13a) and (4.8a) we have $\mathrm{H}_{\varepsilon}=-\varepsilon \partial_{t} Q_{\varepsilon}: \frac{\nabla Q_{\varepsilon}}{\left|\nabla Q_{\varepsilon}\right|}$. Using this, we can expand the integrand in the above estimate and apply the Cauchy-Schwarz inequality to obtain

$$
\begin{aligned}
& \varepsilon^{2}\left|\partial_{t} Q_{\varepsilon}\right|^{2}-\left|\mathrm{H}_{\varepsilon}\right|^{2}+\left.\left|\mathrm{H}_{\varepsilon}-\varepsilon \mathrm{H}_{I}\right| \nabla Q_{\varepsilon}\right|^{2} \\
= & \varepsilon^{2}\left|\partial_{t} Q_{\varepsilon}\right|^{2}+\varepsilon^{2}\left|\mathrm{H}_{I}\right|^{2}\left|\nabla Q_{\varepsilon}\right|^{2}+2 \varepsilon^{2}\left(\mathrm{H}_{I} \cdot \nabla\right) Q_{\varepsilon}: \partial_{t} Q_{\varepsilon} \\
\geqq & \varepsilon^{2}\left|\partial_{t} Q_{\varepsilon}\right|^{2}+\varepsilon^{2}\left|\left(\mathrm{H}_{I} \cdot \nabla\right) Q_{\varepsilon}\right|^{2}+2 \varepsilon^{2}\left(\mathrm{H}_{I} \cdot \nabla\right) Q_{\varepsilon}: \partial_{t} Q_{\varepsilon} \\
= & \varepsilon^{2}\left|\partial_{t} Q_{\varepsilon}+\left(\mathrm{H}_{I} \cdot \nabla\right) Q_{\varepsilon}\right|^{2} .
\end{aligned}
$$

This implies

$$
\int_{0}^{T} \int_{\Omega}\left|\partial_{t} Q_{\varepsilon}+\left(\mathrm{H}_{I} \cdot \nabla\right) Q_{\varepsilon}\right|^{2} \mathrm{~d} x \mathrm{~d} t \leqq e^{(1+T) C\left(I_{0}\right)},
$$

so combining (5.2) with (5.7) leads us to (5.3).

With the above uniform estimates, we can prove the following convergence result:

Proposition 5.2. There exists a subsequence of $\varepsilon_{k}>0$ such that

$$
\begin{aligned}
& {\left[\partial_{t} Q_{\varepsilon_{k}}, Q_{\varepsilon_{k}}\right]=\left[\partial_{t} Q_{\varepsilon_{k}}-\Pi_{Q_{\varepsilon_{k}}} \partial_{t} Q_{\varepsilon_{k}}, Q_{\varepsilon_{k}}\right]} \\
& \stackrel{k \rightarrow \infty}{\longrightarrow} \bar{S}_{0}(x, t) \text { weakly in } L^{2}\left(0, T ; L^{2}(\Omega)\right), \\
& {\left[\partial_{i} Q_{\varepsilon_{k}}, Q_{\varepsilon_{k}}\right]=\left[\partial_{i} Q_{\varepsilon_{k}}-\Pi_{Q_{\varepsilon_{k}}} \partial_{i} Q_{\varepsilon_{k}}, Q_{\varepsilon_{k}}\right]} \\
& \stackrel{k \rightarrow \infty}{\longrightarrow} \bar{S}_{i}(x, t) \text { weakly-star in } L^{\infty}\left(0, T ; L^{2}(\Omega)\right)
\end{aligned}
$$

for $1 \leqq i \leqq d$. Moreover,

$$
\begin{gathered}
\partial_{t} Q_{\varepsilon_{k}} \stackrel{k \rightarrow \infty}{\longrightarrow} \partial_{t} Q, \text { weakly in } L^{2}\left(0, T ; L_{l o c}^{2}\left(\Omega^{ \pm}(t)\right)\right), \\
\nabla Q_{\varepsilon_{k}} \stackrel{k \rightarrow \infty}{\longrightarrow} \nabla Q, \text { weakly in } L^{\infty}\left(0, T ; L_{l o c}^{2}\left(\Omega^{ \pm}(t)\right)\right), \\
Q_{\varepsilon_{k}} \stackrel{k \rightarrow \infty}{\longrightarrow} Q, \text { strongly in } C\left([0, T] ; L_{l o c}^{2}\left(\Omega^{ \pm}(t)\right)\right),
\end{gathered}
$$

where $Q=Q(x, t)$ is represented as

$$
Q(x, t)=s^{ \pm}\left(\mathrm{u}(x, t) \otimes \mathrm{u}(x, t)-\frac{1}{3} I_{3}\right) \text { a.e. }(x, t) \in \Omega_{T}^{ \pm}
$$

for some unit vector field

$$
\begin{aligned}
\mathrm{u} \in L^{\infty}\left(0, T ; H^{1}\left(\Omega^{+}(t) ; \mathbb{S}^{2}\right)\right) & \cap H^{1}\left(0, T ; L^{2}\left(\Omega^{+}(t) ; \mathbb{S}^{2}\right)\right) \\
& \cap C\left([0, T] ; L^{2}\left(\Omega^{+}(t) ; \mathbb{S}^{2}\right)\right) .
\end{aligned}
$$


Proof. We first deduce from (1.6) and (2.10) that $d^{F}(Q)$ is an isotropic function, which only depends on the eigenvalue of $Q \in \mathcal{Q}$. So by (2.8b), the mollified distance function $d_{\varepsilon}^{F}(Q)$ is isotropic and smooth in $Q$. By [4] there exists a smooth symmetric function $g\left(\lambda_{1}, \lambda_{2}, \lambda_{3}\right)$ such that $d_{\varepsilon}^{F}(Q)=g\left(\lambda_{1}(Q), \lambda_{2}(Q), \lambda_{3}(Q)\right)$. Let $Q_{0} \in \mathcal{Q}$ be a matrix having distinct eigenvalues, then $\lambda_{i}(Q)$ as well as the eigenvectors $\mathrm{n}_{i}(Q)$ are real-analytic functions of $Q$ near $Q_{0}$, and then by chain rule

$$
\begin{aligned}
& \frac{\partial d_{\varepsilon}^{F}(Q)}{\partial Q}=\sum_{k=1}^{3} \frac{\partial g}{\partial \lambda_{k}} \frac{\partial \lambda_{k}}{\partial Q}=\sum_{k=1}^{3} \frac{\partial g}{\partial \lambda_{k}} \mathrm{n}_{k}(Q) \otimes \mathrm{n}_{k}(Q), \\
& \text { in a neighborhood of } Q_{0} .
\end{aligned}
$$

In a neighborhood of $Q_{0}$, we also have $Q=\sum_{k=1}^{3} \lambda_{k}(Q) \mathrm{n}_{k}(Q) \otimes \mathrm{n}_{k}(Q)$. So we have

$$
\left[\nabla_{q} d_{\varepsilon}^{F}(Q), Q\right]=0
$$

holds in a neighborhood of $Q_{0}$ having distinct eigenvalues, and thus for every $Q \in \mathcal{Q}$ by continuity. Now in view of (4.10), we have

$$
\begin{aligned}
& {\left[\Pi_{Q_{\varepsilon}} \partial_{t} Q_{\varepsilon}(x, t), Q_{\varepsilon}(x, t)\right]=0,} \\
& {\left[\Pi_{Q_{\varepsilon}} \partial_{i} Q_{\varepsilon}(x, t), Q_{\varepsilon}(x, t)\right]=0 \text { a.e. }(x, t) \in \Omega_{T}}
\end{aligned}
$$

for $1 \leqq i \leqq d$. This together with (3.29) and (5.1) implies

$$
\begin{aligned}
& \left\|\left[\partial_{t} Q_{\varepsilon}, Q_{\varepsilon}\right]\right\|_{L^{2}\left(0, T ; L^{2}(\Omega)\right)}+\left\|\left[\nabla Q_{\varepsilon}, Q_{\varepsilon}\right]\right\|_{L^{\infty}\left(0, T ; L^{2}(\Omega)\right)} \\
& =\left\|\left[\partial_{t} Q_{\varepsilon}-\Pi_{Q_{\varepsilon}} \partial_{t} Q_{\varepsilon}, Q_{\varepsilon}\right]\right\|_{L^{2}\left(0, T ; L^{2}(\Omega)\right)} \\
& \quad+\left\|\left[\nabla Q_{\varepsilon}-\Pi_{Q_{\varepsilon}} \nabla Q_{\varepsilon}, Q_{\varepsilon}\right]\right\|_{L^{\infty}\left(0, T ; L^{2}(\Omega)\right)} \leqq C
\end{aligned}
$$

for some $C$ independent of $\varepsilon$. Combining this estimate with weak compactness implies (5.8).

It follows from (5.2), (5.3), (3.29), and the Aubin-Lions lemma that, for any $\delta>0$, there exists a subsequence $\varepsilon_{k}=\varepsilon_{k}(\delta)>0$ such that

$$
\begin{gathered}
\partial_{t} Q_{\varepsilon_{k}} \stackrel{k \rightarrow \infty}{\longrightarrow} \partial_{t} \bar{Q}_{\delta}, \text { weakly in } L^{2}\left(0, T ; L^{2}\left(\Omega^{ \pm}(t) \backslash I_{t}(\delta)\right)\right), \\
\nabla Q_{\varepsilon_{k}} \stackrel{k \rightarrow \infty}{\longrightarrow} \nabla \bar{Q}_{\delta}, \text { weakly-star in } L^{\infty}\left(0, T ; L^{2}\left(\Omega^{ \pm}(t) \backslash I_{t}(\delta)\right)\right), \\
Q_{\varepsilon_{k}} \stackrel{k \rightarrow \infty}{\longrightarrow} \bar{Q}_{\delta}, \text { weakly-star in } L^{\infty}(\Omega \times(0, T)), \\
Q_{\varepsilon_{k}} \stackrel{k \rightarrow \infty}{\longrightarrow} \bar{Q}_{\delta}, \text { strongly in } C\left([0, T] ; L^{2}\left(\Omega^{ \pm}(t) \backslash I_{t}(\delta)\right)\right) .
\end{gathered}
$$

By a diagonal argument, we infer there exists

$$
Q \in L^{2}\left(0, T ; H_{l o c}^{1}\left(\Omega^{ \pm}(t)\right)\right) \cap L^{\infty}\left(\Omega^{ \pm}\right) \text {, with } \partial_{t} Q \in L^{2}\left(0, T ; L_{l o c}^{2}\left(\Omega^{ \pm}(t)\right)\right)
$$

such that the convergence (5.9) as well as

$$
Q(x, t)=\bar{Q}_{\delta}(x, t) \text { in } L^{\infty}\left(0, T ; H^{1}\left(\Omega^{ \pm}(t) \backslash I_{t}(\delta)\right)\right.
$$


hold for every $\delta>0$ and every $t \in[0, T]$. Moreover, by (5.17), the interpolation theory and $(5.16 \mathrm{c})$, we have

$$
Q \in C\left([0, T] ; L^{2}\left(\Omega^{ \pm}(t)\right) \cap L^{\infty}(\Omega \times(0, T)) .\right.
$$

To prove (5.10), we first deduce that $F(Q)$ has the same regularity as $Q$ in (5.17), and thus by interpolation theory we obtain

$$
F(Q) \in C\left([0, T] ; L^{2}\left(\Omega^{ \pm}(t)\right) .\right.
$$

We use (5.9c), (5.2), and Fatou's lemma to deduce that

$$
F(Q(x, t))=0, \forall t \in[0, T] \text { and a.e. in } x \in \Omega^{ \pm}(t) .
$$

This, together with (1.10), implies

$$
|Q|(x, t) \in\left\{0, s^{+} \sqrt{\frac{2}{3}}\right\}, \forall t \in[0, T] \text { and a.e. in } x \in \Omega^{ \pm}(t) .
$$

By taking the $L^{2}$-norm, we obtain two continuous functions:

$$
f^{ \pm}(t):=\|Q(\cdot, t)\|_{L^{2}\left(\Omega^{ \pm}(t)\right)} \in C\left([0, T] ;\left\{0, s^{+} \sqrt{\frac{2}{3}\left|\Omega^{ \pm}(t)\right|}\right\}\right) .
$$

On the other hand, by the choice of the initial condition (2.21) and the convergence (5.16d), we deduce that

$$
Q(x, 0)=\mathbf{1}_{\Omega^{+}(0)} s^{+}\left(\mathrm{u}^{i n}(x) \otimes \mathrm{u}^{i n}(x)-\frac{1}{3} I_{3}\right), \text { a.e. in } \Omega^{ \pm}(0) \backslash I_{0}(\delta)
$$

for any $\delta>0$ and thus for $\delta=0$. This implies $f^{+}(0)=s^{+} \sqrt{\frac{2}{3}\left|\Omega^{+}(0)\right|}, f^{-}(0)=0$ and thus

$$
f^{+}(t)=s^{+} \sqrt{\frac{2}{3}\left|\Omega^{+}(t)\right|}, f^{-}(t)=0, \quad \forall t \in[0, T]
$$

This, together with (5.19), implies

$$
\begin{gathered}
Q(x, t)=0, \forall t \in[0, T] \text { and a.e. in } x \in \Omega^{-}(t), \\
Q(x, t) \in \mathcal{N}, \forall t \in[0, T] \text { and a.e. in } x \in \Omega^{+}(t),
\end{gathered}
$$

and thus (5.10) is proved.

By (5.10), (5.17), and the orientability theorem by Ball-Zarnescu [6, Section 3.2] implies that $Q$ is uniaxial (5.10) for some

$$
\mathrm{u} \in L^{\infty}\left(0, T ; H_{l o c}^{1}\left(\Omega^{+}(t) ; \mathbb{S}^{2}\right)\right) \text { with } \partial_{t} \mathrm{u} \in L^{2}\left(0, T ; L_{l o c}^{2}\left(\Omega^{+}(t) ; \mathbb{S}^{2}\right)\right) .
$$

It remains to improve the integrability of $\nabla_{x, t} \mathrm{u}$. To this end, we choose a sequence

$$
\psi_{\ell}(x, t) \in C_{c}^{\infty}\left(\Omega_{T}^{+}\right) \text {such that } \psi_{\ell}(x, t) \stackrel{\ell \rightarrow \infty}{\longrightarrow} \mathbf{1}_{\Omega_{T}^{+}}(x, t) .
$$


Since $|\mathrm{u}|=1$ a.e., by (5.8a), (5.8b) and (5.9), we deduce that for almost every $(x, t) \in \Omega_{T}^{+}$, it holds that

$$
\psi_{\ell} \bar{S}_{i}=\psi_{\ell}\left[\partial_{i} Q, Q\right]=s_{+}^{2} \psi_{\ell}\left(\partial_{i} \mathrm{u} \otimes \mathrm{u}-\mathrm{u} \otimes \partial_{i} \mathrm{u}\right), 0 \leqq i \leqq d,
$$

where $\partial_{0}:=\partial_{t}$. Note that for each fixed $i \in\{0, \ldots, 3\}$,

$$
\partial_{i} \mathrm{u} \otimes \mathrm{u}-\mathrm{u} \otimes \partial_{i} \mathrm{u}=\left(\begin{array}{ccc}
0 & \left(\partial_{i} \mathrm{u} \wedge \mathrm{u}\right)_{3} & -\left(\partial_{i} \mathrm{u} \wedge \mathrm{u}\right)_{2} \\
-\left(\partial_{i} \mathrm{u} \wedge \mathrm{u}\right)_{3} & 0 & \left(\partial_{i} \mathrm{u} \wedge \mathrm{u}\right)_{1} \\
\left(\partial_{i} \mathrm{u} \wedge \mathrm{u}\right)_{2} & -\left(\partial_{i} \mathrm{u} \wedge \mathrm{u}\right)_{1} & 0
\end{array}\right)
$$

where $\left(\partial_{i} \mathrm{u} \wedge \mathrm{u}\right)_{k}$ denotes the $k$-th component of the 3 -vector $\partial_{i} \mathrm{u} \wedge \mathrm{u}$. Since $\bar{S}_{i}$ are $L^{2}$ integrable in $\Omega_{T}$, sending $\ell \rightarrow \infty$ and applying the dominated convergence theorem to the above identity lead us to

$$
\begin{aligned}
& \partial_{t} \mathrm{u} \wedge \mathrm{u} \in L^{\infty}\left(0, T ; L^{2}\left(\Omega^{+}(t)\right)\right), \\
& \partial_{i} \mathrm{u} \wedge \mathrm{u} \in L^{2}\left(0, T ; L^{2}\left(\Omega^{+}(t)\right)\right), \text { for } i \in\{1, \ldots, d\} .
\end{aligned}
$$

Retaining that $\mathrm{u}$ maps into $\mathbb{S}^{2}$, we deduce

$$
\left|\partial_{t} \mathrm{u}\right|^{2}=\left|\partial_{t} \mathrm{u} \wedge \mathrm{u}\right|^{2}, \quad\left|\partial_{i} \mathrm{u}\right|^{2}=\left|\partial_{i} \mathrm{u} \wedge \mathrm{u}\right|^{2} \text { a.e. in } \Omega_{T}^{+}, 1 \leqq i \leqq d,
$$

so we improve (5.22) to (5.11).

Proof of Theorem 2.1. In the course of the proof, we shall adopt the notation $A$ : $B=\operatorname{tr} A^{T} B$ for any $A, B \in \mathbb{R}^{3 \times 3}$. We associate each testing vector field $\varphi(x, t)=$ $\left(\varphi_{1}, \varphi_{2}, \varphi_{3}\right) \in C^{1}\left(\overline{\Omega_{T}}, \mathbb{R}^{3}\right)$ a matrix-valued function

$$
\Phi(x, t)=\left(\begin{array}{ccc}
0 & \varphi_{3} & -\varphi_{2} \\
-\varphi_{3} & 0 & \varphi_{1} \\
\varphi_{2} & -\varphi_{1} & 0
\end{array}\right)
$$

Since $\left[\nabla_{q} F\left(Q_{\varepsilon_{k}}\right), Q_{\varepsilon_{k}}\right]=0$, applying the anti-symmetric product $\left[\cdot, Q_{\varepsilon_{k}}\right]$ to (1.13a) and integration by parts over $\Omega_{T}$ yields

$$
\int_{\Omega_{T}}\left[\partial_{t} Q_{\varepsilon_{k}}, Q_{\varepsilon_{k}}\right]: \Phi \mathrm{d} x \mathrm{~d} t+\int_{\Omega_{T}} \sum_{j=1}^{3}\left[\partial_{j} Q_{\varepsilon_{k}}, Q_{\varepsilon_{k}}\right]: \partial_{j} \Phi \mathrm{d} x \mathrm{~d} t=0 .
$$

Note that no boundary integral will occur due to $(1.13 \mathrm{c})$. Recall that we denote $I_{t}(\delta)$ the $\delta$ - neighborhood of $I_{t}$. Equivalently, we can write the above equation by

$$
\begin{aligned}
& \sum_{ \pm} \int_{0}^{T} \int_{\Omega^{ \pm}(t) \backslash I_{t}(\delta)}\left(\left[\partial_{t} Q_{\varepsilon_{k}}, Q_{\varepsilon_{k}}\right]: \Phi+\sum_{j=1}^{3}\left[\partial_{j} Q_{\varepsilon_{k}}, Q_{\varepsilon_{k}}\right]: \partial_{j} \Phi\right) \mathrm{d} x \mathrm{~d} t \\
& +\int_{0}^{T} \int_{I_{t}(\delta)}\left(\left[\partial_{t} Q_{\varepsilon_{k}}, Q_{\varepsilon_{k}}\right]: \Phi+\sum_{j=1}^{3}\left[\partial_{j} Q_{\varepsilon_{k}}, Q_{\varepsilon_{k}}\right]: \partial_{j} \Phi\right) \mathrm{d} x \mathrm{~d} t=0 .
\end{aligned}
$$


Using (5.9), (5.8) and (5.10), we can pass $k \rightarrow \infty$ and yield

$$
\begin{gathered}
\int_{0}^{T} \int_{\Omega^{+}(t) \backslash I_{t}(\delta)}\left(\left[\partial_{t} Q, Q\right]: \Phi+\sum_{j=1}^{3}\left[\partial_{j} Q, Q\right]: \partial_{j} \Phi\right) \mathrm{d} x \mathrm{~d} t \\
\quad+\int_{0}^{T} \int_{I_{t}(\delta)}\left(\bar{S}_{0}: \Phi+\sum_{j=1}^{3} \bar{S}_{j}: \partial_{j} \Phi\right) \mathrm{d} x \mathrm{~d} t=0 .
\end{gathered}
$$

By $|\mathrm{u}|=1$ a.e., (5.10), (5.27) and (5.25), we obtain the following identities :

$$
\begin{gathered}
{\left[\partial_{t} Q, Q\right]: \Phi=s_{+}^{2}\left(\partial_{t} \mathrm{u} \otimes \mathrm{u}-\mathrm{u} \otimes \partial_{t} \mathrm{u}\right): \Phi=2 s_{+}^{2} \partial_{t} \mathrm{u} \wedge \mathrm{u} \cdot \varphi} \\
{\left[\partial_{j} Q, Q\right]: \partial_{j} \Phi=s_{+}^{2}\left(\partial_{j} \mathrm{u} \otimes \mathrm{u}-\mathrm{u} \otimes \partial_{j} \mathrm{u}\right): \partial_{j} \Phi=2 s_{+}^{2} \partial_{j} \mathrm{u} \wedge \mathrm{u} \cdot \partial_{j} \varphi}
\end{gathered}
$$

Thus we obtain

$$
\begin{gathered}
2 s_{+}^{2} \int_{0}^{T} \int_{\Omega^{+}(t) \backslash I_{t}(\delta)}\left(\partial_{t} \mathrm{u} \wedge \mathrm{u} \cdot \varphi+\sum_{j=1}^{3}\left(\partial_{j} \mathrm{u} \wedge \mathrm{u}\right) \cdot \partial_{j} \varphi\right) \mathrm{d} x \mathrm{~d} t \\
\quad+\int_{0}^{T} \int_{I_{t}(\delta)}\left(\bar{S}_{0}: \Phi+\sum_{j=1}^{3} \bar{S}_{j}: \partial_{j} \Phi\right) \mathrm{d} x \mathrm{~d} t=0 .
\end{gathered}
$$

Due to (5.11) we have the absolute continuity of $\partial_{t} \mathrm{u} \wedge \mathrm{u}$ and $\nabla \mathrm{u} \wedge \mathrm{u}$ in $\Omega_{T}^{+}$. Moreover, (5.8) implies the absolute continuity of $\left\{\bar{S}_{i}\right\}_{0 \leqq i \leqq d}$ in $\Omega_{T}$. So we can pass to the limit $\delta \rightarrow 0$ in the above identity, which yields

$$
\int_{0}^{T} \int_{\Omega^{+}(t)} \partial_{t} \mathrm{u} \wedge \mathrm{u} \cdot \varphi \mathrm{d} x \mathrm{~d} t+\int_{0}^{T} \int_{\Omega^{+}(t)} \sum_{j=1}^{3}\left(\partial_{j} \mathrm{u} \wedge \mathrm{u}\right) \cdot \partial_{j} \varphi \mathrm{d} x \mathrm{~d} t=0
$$

This concludes the proof of Theorem 2.1.

Acknowledgements. T. Laux is funded by the Deutsche Forschungsgemeinschaft (DFG, German Research Foundation) under Germany's Excellence Strategy - EXC-2047/1 - 390685813. Y. Liu is partially supported by NSF of China under Grant 11971314.

Funding Open Access funding enabled and organized by Projekt DEAL.

Open Access This article is licensed under a Creative Commons Attribution 4.0 International License, which permits use, sharing, adaptation, distribution and reproduction in any medium or format, as long as you give appropriate credit to the original author(s) and the source, provide a link to the Creative Commons licence, and indicate if changes were made. The images or other third party material in this article are included in the article's Creative Commons licence, unless indicated otherwise in a credit line to the material. If material is not included in the article's Creative Commons licence and your intended use is not permitted by statutory regulation or exceeds the permitted use, you will need to obtain permission directly from the copyright holder. To view a copy of this licence, visit http://creativecommons.org/ licenses/by/4.0/.

Publisher's Note Springer Nature remains neutral with regard to jurisdictional claims in published maps and institutional affiliations. 


\section{References}

1. AlPER, O.: Rectifiability of line defects in liquid crystals with variable degree of orientation. Arch. Ration. Mech. Anal. 228(1), 309-339, 2018

2. Alper, O., Hardt, R., Lin, F.-H.: Defects of liquid crystals with variable degree of orientation. Calc. Var. Partial Differ. Equ., 56(5):Paper No. 128, 32, 2017.

3. Ambrosio, L., Dal Maso, G.: A general chain rule for distributional derivatives. Proc. Am. Math. Soc. 108(3), 691-702, 1990

4. BALl, J.M.: Differentiability properties of symmetric and isotropic functions. Duke Math. J. 51(3), 699-728, 1984

5. BALl, J.M., Majumdar, A.: Nematic liquid crystals: From Maier-Saupe to a continuum theory. Mol. Cryst. Liq. Cryst. 525(1), 1-11, 2010

6. BAll, J.M., ZARnesCu, A.: Orientability and energy minimization in liquid crystal models. Arch. Ration. Mech. Anal. 202(2), 493-535, 2011

7. Bedford, S.: Function spaces for liquid crystals. Arch. Ration. Mech. Anal. 219(2), 937-984, 2016

8. De Gennes, P.G., Prost, J.: The Physics of Liquid Crystals, 2nd edn. International Series of Monographs on Physics. Oxford University Press, Incorporated 1995

9. Ericksen, J.L.: Liquid crystals with variable degree of orientation. Arch. Ration. Mech. Anal. 113(2), 97-120, 1990

10. Fei, M., Wang, W., Zhang, P., Zhang, Z.: Dynamics of the nematic-isotropic sharp interface for the liquid crystal. SIAM J. Appl. Math. 75(4), 1700-1724, 2015

11. FeI, M., WANG, W., Zhang, P., Zhang, Z.: On the isotropic-nematic phase transition for the liquid crystal. Peking Math. J. 1(2), 141-219, 2018

12. Fischer, J., Laux, T., Simon, T.M.: Convergence rates of the Allen-Cahn equation to mean curvature flow: a short proof based on relative entropies. SIAM J. Math. Anal. 52(6), 6222-6233, 2020

13. Fonseca, I., TARTAR, L.: The gradient theory of phase transitions for systems with two potential wells. Proc. R. Soc. Edinb. Sect. A 111(1-2), 89-102, 1989

14. Golovaty, D., Novack, M., Sternberg, P., Venkatraman, R.: A model problem for nematic-isotropic transitions with highly disparate elastic constants. Arch. Ration. Mech. Anal. 236(3), 1739-1805, 2020

15. Golovaty, D., Sternberg, P., Venkatraman, R.: A Ginzburg-Landau-type problem for highly anisotropic nematic liquid crystals. SIAM J. Math. Anal. 51(1), 276-320, 2019

16. Jerrard, R.L., Smets, D.: On the motion of a curve by its binormal curvature. J. Eur. Math. Soc. (JEMS) 17(6), 1487-1515, 2015

17. Laux, T., Simon, T.M.: Convergence of the Allen-Cahn equation to multiphase mean curvature flow. Commun. Pure Appl. Math. 71(8), 1597-1647, 2018

18. Lin, F.-H.: On nematic liquid crystals with variable degree of orientation. Commun. Pure Appl. Math. 44(4), 453-468, 1991

19. Lin, F.-H., PAN, X.-B., WANG, C.-Y.: Phase transition for potentials of high-dimensional wells. Commun. Pure Appl. Math. 65(6), 833-888, 2012

20. Lin, F.-H.; Poon, C.: On Ericksen's model for liquid crystals. J. Geom. Anal. 4(3), 379-392, 1994

21. Lin, F.-H., WANG, C.-Y.: Harmonic maps in connection of phase transitions with higher dimensional potential wells. Chin. Ann. Math. Ser. B 40(5), 781-810, 2019

22. Lin, F.-H., WANG, C.-Y.: Isotropic-nematic phase transition and liquid crystal droplets. arXiv preprint arXiv:2009.11487, 2020

23. Majumdar, A., Zarnescu, A.: Landau-De Gennes theory of nematic liquid crystals: the Oseen-Frank limit and beyond. Arch. Ration. Mech. Anal. 196(1), 227-280, 2010

24. Park, J., Wang, W., Zhang, P., Zhang, Z.: On minimizers for the isotropic-nematic interface problem. Calc. Var. Partial Differ. Equ., 56(2):Paper No. 41, 15, 2017

25. Rubinstein, J., Sternberg, P., Keller, J.B.: Fast reaction, slow diffusion, and curve shortening. SIAM J. Appl. Math. 49(1), 116-133, 1989 
26. Sternberg, P.: The effect of a singular perturbation on nonconvex variational problems. Arch. Ration. Mech. Anal. 101(3), 209-260, 1988

\section{TIM LaUX}

Hausdorff Center for Mathematics, University of Bonn,

Villa Maria, Endenicher Allee 62, 53115 Bonn

Germany.

e-mail: tim.laux@hcm.uni-bonn.de

and

YUNING LIU

NYU Shanghai,

1555 Century Avenue,

Shanghai

200122 China.

e-mail: yl67@nyu.edu

and

NYU-ECNU Institute of Mathematical Sciences at NYU Shanghai, 3663 Zhongshan Road North,

Shanghai

200062 China.

(Received October 19, 2020 / Accepted May 21, 2021)

Published online June 28, 2021

(C) The Author(s) (2021) 